\title{
Clustering By Academic Major at Historically Black Colleges and Universities (HBCUs)
}

\author{
Aaron Goodson
}

Follow this and additional works at: https://researchrepository.wvu.edu/etd

\section{Recommended Citation}

Goodson, Aaron, "Clustering By Academic Major at Historically Black Colleges and Universities (HBCUs)" (2015). Graduate Theses, Dissertations, and Problem Reports. 5696.

https://researchrepository.wvu.edu/etd/5696

This Thesis is protected by copyright and/or related rights. It has been brought to you by the The Research Repository @ WVU with permission from the rights-holder(s). You are free to use this Thesis in any way that is permitted by the copyright and related rights legislation that applies to your use. For other uses you must obtain permission from the rights-holder(s) directly, unless additional rights are indicated by a Creative Commons license in the record and/ or on the work itself. This Thesis has been accepted for inclusion in WVU Graduate Theses, Dissertations, and Problem Reports collection by an authorized administrator of The Research Repository @ WVU. For more information, please contact researchrepository@mail.wvu.edu. 
Clustering By Academic Major at Historically Black Colleges and Universities (HBCUs)

\title{
Aaron Goodson
}

Thesis submitted to the College of Physical Activity and Sport Sciences at West Virginia University in partial fulfillment of the requirements for the degree of

\author{
Master of Science \\ in \\ Sport and Exercise Psychology \\ West Virginia University \\ Dana Brooks, Ed.D., Chair \\ Jack Watson II, Ph.D. \\ Ed Jacobs, Ph.D. \\ Department of Sport Sciences \\ Morgantown, WV
}

2015

Keywords: black student-athletes, $\mathrm{HBCU}$, academic major, clustering 


\begin{abstract}
Clustering By Academic Major at Historically Black Colleges and Universities (HBCUs)
\end{abstract}

\title{
Aaron Goodson
}

Follow-up studies of clustering by academic major, the dynamic of $25 \%$ or more of the studentathletes on a roster pursuing the same academic major, indicate that it still occurs in revenuegenerating sports (Fountain \& Finley 2009, 2011; Otto, 2012). Clustering challenges the notion that student-athletes have control over their collegiate academic experience and reveals that their educational pursuits may not align with their professional goals or provide a meaningful educational experience (Sharp \& Sheilley, 2008). Research on clustering is absent in member institutions of different NCAA divisions, institutions with unique missions and history (such as Historically Black Colleges and Universities (HBCUs) or single-sex institutions), and institutions of higher education in other organizations such as junior colleges or community colleges. This study examined the academic majors of student-athletes in football and men's basketball at select HBCUs over four years. The results revealed that clustering occurred within basketball at four institutions, but only occurred within football at one institution. Additionally, clustering by academic major occurred at NCAA Division I and II institutions. From these results, it is clear that clustering by academic major is not just an issue at "big time" institutions, but potentially a widespread issue across collegiate athletics. 
Table of Contents

$\begin{array}{ll}\text { Introduction } & 1\end{array}$

$\begin{array}{lr}\text { Methodology } & 8\end{array}$

$\begin{array}{ll}\text { Pilot Research } & 8\end{array}$

$\begin{array}{ll}\text { Research Design } & 10\end{array}$

Research Questions and Hypotheses $\quad 11$

$\begin{array}{ll}\text { Sampling/Recruitment } & 12\end{array}$

Measures, Variables, \& Procedure 13

$\begin{array}{ll}\text { Results } & 14\end{array}$

$\begin{array}{ll}\text { Ad Hoc Analyses } & 17\end{array}$

$\begin{array}{ll}\text { Discussion } & 19\end{array}$

$\begin{array}{ll}\text { References } & 25\end{array}$

$\begin{array}{ll}\text { Appendix A - Tables } & 30\end{array}$

Table 1: Mid-Eastern Athletic Conference Football Academic Major 30

Table 2: Central Intercollegiate Athletic Association Football Academic Major 31

Table 3: Mid-Eastern Athletic Conference Basketball Academic Major 32

Table 4: Central Intercollegiate Athletic Association Basketball Academic Major 33

Table 5: Mid-Eastern Athletic Conference General Student Population 34

Table 6: Central Intercollegiate Athletic Conference General Student Population 35

Table 7: Most Common Academic Majors Among Student-Athletes 36

Table 8: Most Common Areas of Study Among Student-Athletes 36

Table 9: Most Common Areas of Study Among Student-Athletes by Conference 36

Table 10: Mid-Eastern Athletic Conference Football Area of Study 37 
Table 11: Central Intercollegiate Athletic Conference Football Area of Study 38

Table 12: Mid-Eastern Athletic Conference Basketball Area of Study 39

Table 13: Central Intercollegiate Athletic Conference Basketball Area of Study $\quad 40$

Appendix B - Extended Review of Literature 41

Appendix C - NCAA GOALS Sorting Sheet + University Majors 87

Appendix D - Academic Majors Coding Sheet by NCAA GOALS 97 
Running Head: Clustering by Academic Major at HBCUs

\section{Introduction}

Recently, infractions and challenges of exploitation have plagued NCAA Division I football and basketball with marquee institutions such as the Syracuse University, University of Notre Dame, University of Connecticut, and the University of North Carolina at Chapel Hill making headlines for cases of academic fraud among the institution's student-athletes (Osborne, 2014). Scholars have analyzed the impact of increased commercialization and the economic model of intercollegiate athletics as the source of the large number of recent infractions and violations (Osborne, 2014; Lanter \& Hawkins, 2013). Although there are penalties for recruiting violations and improper benefits for student-athletes, many of the scandals in intercollegiate athletics have stemmed from academic eligibility concerns surrounding student-athletes. To address many of these concerns through the years, the National Collegiate Athletic Association (NCAA) has passed legislation that holds member institutions accountable for the academic experience of the student-athletes that represent the institution. NCAA academic policy has grown and changed several times in the last four decades. The focus of early policy was studentathlete initial eligibility, but today the focus of policies is student-athlete graduation rates. Many of the NCAA promotional commercials during televised competitions address the student-athlete experience outside of sport by stating, "Many of them [student-athletes] will go pro in something other than sports (NCAA, n.d.)." There are many college experiences that affect the trajectory of student-athletes' careers, and one of the most significant influences on that trajectory is a student-athlete's academic major.

During the opening minutes of a college football game or in slower moments such as free throw attempts in college basketball games, the network broadcasting crew shares the studentathlete's major with its viewers (Suggs, 2003). The recognition of the number of similar majors 
among players or unique majors from non-athlete student majors can stir different responses in viewers. Some viewers may observe that a substantial number of student-athletes on the same team pursue the same major. Other viewers may notice that some student-athletes have a major in an area of study that is seemingly not challenging or an area of study that is uncommon in higher education. In the past, these observations have led to an informal debate about the challenges, or lack thereof, in the intercollegiate student-athlete academic experience. As long as student-athletes are graduating, the NCAA can promote student-athlete academic success, but there is research that indicates the differences in the potential earnings of college graduates as dictated by their academic major (Sanders \& Hildenbrand, 2010; PayScale, 2015). NCAA student-athletes at Divisions I and II are eligible for athletic financial aid (scholarships) that pay their tuition and fees, housing, and most other expenses. In recent debates about whether studentathletes should be compensated for their efforts, some argue against the notion by stating that athletic financial aid is sufficient compensation and that student-athletes have free will to determine the quality of their academic experience once they gain admission to the institution. In fact, some student-athletes may not otherwise be eligible to attend the institution without their scholarship and commitment to compete for the university. At some institutions, coaches and athletic department administrators have received financial bonuses for teams exceeding APR and graduation rate standards (Berkowitz et al., 2013).

Intercollegiate student-athletes represent a special population of college students in the United States. Intercollegiate student-athletes adhere to a set of expectations different from other college students and have a different college experience from other college students (Melendez, 2010). It is widely known that student-athletes sacrifice a large portion of their time spent in college for sport participation with practices, games, traveling, and other obligations (Sharp \& 
Sheilley, 2008). Therefore, it is important that student-athletes find effective ways to manage the obligations that come with their athletic participation while fulfilling their obligations as a student. With regard to pursuing certain academic majors or seizing opportunities that enhance a student's academic experience during their time in college, NCAA student-athletes' time obligations often prohibit them from taking certain classes, and therefore, pursuing certain academic majors, or seizing these aforementioned opportunities.

Clustering by academic major can be seen as one of the ways that student-athletes balance the demands of being a student and competing as an athlete for their college or university (Steeg, 2008). It is believed that student-athletes cluster in academic majors that are less rigorous than others to maintain their eligibility and achieve academic success while dedicating satisfactory time and energy to their sport (Steeg, 2008). Clustering by academic major has been operationally defined as the phenomenon that exists when $25 \%$ or more of the student-athletes on a team pursue the same academic major and that the percentage of studentathletes on a team in this major exceeds the percentage of the general student body pursuing the same academic major (Case, Greer, \& Brown, 1987). Investigations of academic clustering have their roots in the study of the student-athlete academic experience, exploitation of the studentathlete (Renick, 1974; Sack, 1986), and NCAA legislation to support the academic endeavors of collegiate student-athletes. Although the seminal study of clustering by academic major (Case, Brown, \& Greer, 1987) was limited to NCAA Division I men's and women's basketball teams, many of the following studies were limited to football (Fountain \& Finley, 2009; Schneider, Ross, \& Fisher, 2010; Fountain \& Finley, 2011). Much of the media coverage and non-scholarly work about clustering has studied NCAA Division I football (Suggs, 2003; Steeg, 2008). Case, Brown, and Greer's (1987) research revealed differences in clustering at institutions with "big 
time" athletic programs (finishing the season with a high national ranking in the last three years) and "elite" academic reputations. Case, Brown, and Greer (1987) asserted that clustering at institutions with "elite" academic reputations may be more pervasive because student-athletes may be at a different academic standard than their non-athlete peers. This gap in the academic performance of student-athletes as compared to their non-athlete peers could lead to special majors being created to house or "dump" student-athletes (p. 51). Fountain and Finley (2009) and Schneider, Ross, and Fisher (2010) conducted research in two of the power five NCAA Division I football conferences, the Big 12 and the Atlantic Coast Conference (ACC). Institutions that compete in the BCS Conferences/College Football Playoff have historically finished in the Associated Press (AP) Top 25 and College Football Playoff Top 25 Rankings at the end of each season more often than institutions that do not compete in the power five conferences, which qualifies many of them as institutions with "big time" athletic programs.

Classifications such as "elite" academic institution and "big time" program seemingly make it easy to exclude some institutions from analyses of clustering. However, these terms are vague and warrant further investigation and exploration. While the majority of Historically Black Colleges and Universities (HBCUs) do not currently finish among or even reach the AP Top 25 in NCAA Division I or II and are ineligible to compete in the College Football Playoff, some HBCUs have historically been considered highly successful athletic and academic undergraduate and graduate programs. In addition to their strong academic and athletic reputation among many black Americans, the unique history and mission, commitment, and current institutional challenges make HBCUs institutions of interest for this study (Nichols, 2004; Kim \& Conrad, 2006; Coupet, 2013; Shropshire, 2013). HBCUs were defined in the 1965 Higher Education act as higher education institutions that were established before 1964 with the mission to educate 
black Americans (Hodge, Bennett III, \& Collins, 2013). In their inception, HBCUs were institutions that combatted the legal segregation of the times (Hodge, Bennett III, \& Collins, 2013). However, now that legal segregation has been banned for more than 50 years, the current role and relevance of HBCUs has been publicly challenged (Cantey, Bland, Mack, \& Joy-Davis, 2011; Bettez \& Suggs, 2012; Brown II, 2013). Regardless of one's opinion on the significance of HBCUs, the fact remains that HBCUs still serve a sizable population of college students (Gasman, 2011; Johnson, 2013). According to the National Center of Education Statistics, there are currently 105 HBCUs that enroll 11\% of black students in the United States (NCES, 2011). College students that attend HBCUs have the opportunities to participate in intercollegiate athletics just like students at predominantly white institutions (PWIs) and student-athletes at HBCUs may face similar challenges. A dearth of research exists about the student-athlete experience at HBCUs just as a dearth of research exists about HBCUs as compared to the amount of research that exists about PWIs.

The general student body makeup of HBCUs provides an intersection between many of the most common populations examined in sport studies - males, black student-athletes, and revenue generating sport student-athletes. Although most HBCUs are members of NCAA Division II, III conferences, or other collegiate sports organizations such as the National Association of Intercollegiate Athletics (NAIA), there are two NCAA Division I conferences comprised entirely of HBCUs - the Mid-Eastern Athletic Conference (MEAC) and the Southern Intercollegiate Athletic Conference (SIAC) (Hodge et. al., 2013; Cooper \& Hawkins, 2012). Therefore, HBCUs provide a space to study black students and black student-athlete experiences across all three NCAA divisions. Although all HBCUs were opened with the distinct mission of providing education to black Americans, HBCUs provide education to students from all 
backgrounds. Recently, student body populations at some HBCUs have reported student body demographics that are no longer overwhelmingly black. According to Gasman (2011), a quarter of today's HBCUs have at least a 20\% non-Black student body. Gasman (2011) also noted that HBCUs had an average graduation rate of $30 \%$ based on NCES statistics in 2011, but it is important to note that the majority of HBCU students are low-income, first-generation and PellGrant-eligible. Research shows that students who meet these classifications are less likely to graduate, regardless of where they attend college (Gasman, 2011). The average six-year graduation rate at public and private four-year HBCUs was $29 \%$ and $32 \%$ respectively, both more than 20 percentage points lower than the national average graduation rate of $55.5 \%$ and at least 5 percentage points lower than the national average for black students graduating (Gasman, 2011).

In 2012, DiverseEducation.com published an article entitled "HBCUs Could Be Hit Hard By New NCAA Rules.” The article outlined concerns raised by several HBCU presidents that the new academic standards that raise minimum requirements to qualify for NCAA competition could negatively impact the student-athletes already competing at the institution and future recruiting (Stuart, 2012). Furthermore, the presidents lamented that an open opposition to the new rules would send the message that athletics is valued more than academics, yet a silent acceptance of the new rules forces the institution to make tough decisions around some high achieving student-athletes that could help lead the team to post-season tournaments, which grant the institution more funding and visibility. According to Stuart (2012), NCAA Division I athletic programs generated more than $\$ 450$ million dollars in the 2010 -2011 season through their radio and telecast programming, game ticket sales, and other revenue, and about $60 \%$ of those funds were distributed to member conferences and institutions. NCAA Division I officials have 
compromised with NCAA Division I HBCUs by giving what they call "limited resource institutions" (LRIs) more time to comply with the new standards. All NCAA Division I HBCUs are considered limited resource institutions.

HBCUs can be considered academically elite institutions and their athletic programs have historically been considered "big time." Yet, these institutions have previously been excluded from investigations of clustering by academic major, despite the fact that student-athletes that compete at these institutions fit the four populations that Case, Brown, and Greer (1987) outlined in the results of their study. Additionally, the fact that some of these institutions at the NCAA Division I level are considered LRIs and are working to comply with new NCAA Division I academic legislation begs the question of how these institutions will be able to comply with these requirements given their limited resources. Clustering by academic major is one way that student-athletes can achieve APR and graduation rate goals.

\section{Study Purposes and Significance}

The purpose of this study was to expand the current scope of research on clustering by academic major. All previous published work about clustering has focused on NCAA Division I football and men's basketball at institutions in the power five conferences. The results of this study revealed whether clustering by academic major was unique to NCAA Division I football and basketball programs or a more common issue that spans across NCAA Divisions and institutions.

This study examined football and men's basketball in two conferences mostly comprised of HBCUs. In 2008, one of the conferences had a university join that was not a HBCU. One of the two conferences competes at the NCAA Division I level, and the other conference competes at the NCAA Division II level. Based on the literature about clustering by academic major and 
the factors that lead to its existence (Case, Greer, \& Brown, 1987), the researcher hypothesized that clustering by academic major would exist at these select HBCUs. The literature also indicates that the academic major(s) that contains a cluster of student-athletes differs by institution (Fountain \& Finley, 2009; Otto, 2012). Previous research has indicated that clustering happened more frequently for male student-athletes than female student-athletes and more frequently for black student-athletes than white student-athletes (Case, Brown, \& Greer, 1987). However, this study was designed to reveal information about academic clustering when the majority or all of the student-athletes on a roster were black males. Student-athletes attending HBCUs are previously unstudied populations in analyses of clustering by academic major. The results of this study revealed information that provides context for the student-athlete academic experience beyond NCAA Division I institutions, predominantly white institutions, and institutions with mainstream visibility.

\section{Pilot Research}

\section{Methodology}

Due to the limited resources at some HBCUs, the researcher was unsure about the availability of media guides from some institutions. Therefore, a pilot study was designed and conducted to examine the feasibility of the current study. In the pilot study, the researcher sought to obtain media guides from select HBCUs over a consistent range of time. Media guides were obtained via the Internet. When media guides had not been published online, the Sports Information Director (SID) from the institution's athletic department was contacted and media guides for the missing seasons were requested. Data for the pilot study was collected in the spring and summer of 2014.

The data in the pilot study was comprised of 574 entries from five select North Carolina HBCUs. Three of the institutions were members of the CIAA, a NCAA Division II conference, 
and the other two institutions were members of the MEAC, a NCAA Division I conference. These institutions were selected because of their long history of academic and athletic excellence in North Carolina, thus satisfying certain conditions under which clustering occurs as outlined by Case, Brown, and Greer (1987). Only student-athletes listed as juniors and seniors (redshirt student-athletes included) were selected for the study, although data existed for some studentathletes listed as freshmen and sophomores. Freshmen and sophomores were excluded from the study because of the possibility that their academic major was undecided or their listed major changed before it was officially declared at the start of their fifth semester (Fountain \& Finley, 2009).

An analysis of the pilot data addressed each of the research questions. First, there were instances of clustering by academic major at some institutions. There were no instances of extreme clustering. Second, there was little to no difference in the rate at which clustering by academic major occurred at NCAA Division I and II HBCUs. Clustering by academic major occurred at one institution in each NCAA Division. Across all five institutions, the highest percentages of junior and senior student-athletes pursued academic majors in sport management, physical education, communications, business, and criminal justice. The researcher conducted another analysis that revealed the percentage of student-athletes who pursued academic majors that were classified under particular areas of study (e.g. Business, Social Sciences, Education). When the analysis was expanded to area of study, the highest percentages of student-athletes were in business majors, exercise, sports, and kinesiology majors, and professional studies majors (physical therapy, criminal justice, etc.). While the data are indicative of patterns and trends in student-athletes' decisions about academic majors and areas of study, it was clear that 
data from more institutions would provide a stronger context and support for the emerging patterns and trends.

The number of student-athletes whose majors were not listed or undecided clouded the data. The researcher decided to expand the scope of the study from upperclassmen (junior and senior student-athletes) to include student-athletes in all academic years. This decision allowed for a larger amount of data and more valid data for analysis.

\section{Research Design}

Researchers have studied clustering by analyzing media guides published in print or on the Internet (Case, Greer, \& Brown, 1987; Fountain \& Finley, 2009; Schneider, Ross, \& Fisher, 2010; Fountain \& Finley, 2011; Otto, 2012). In one study of clustering, the researchers (Case et. al, 1987) also sent a questionnaire to department chairpersons where clustering had occurred, in order to compare their findings to the rates at which the general student body population pursued the particular academic majors. Steeg (2008) extended the operational definition of clustering and established the term 'extreme clustering', which occurs when $40 \%$ or more of the studentathletes on a team pursue the same academic major. The researcher denoted clustering by the traditional definition and extreme clustering. In addition to investigating clustering by academic major, this study organized student-athlete academic majors into overarching areas of study using the answer choices from a question on the NCAA Growth Opportunities and Learning Strategies (GOALS) Questionnaire that student-athletes complete yearly to streamline analyses and comparisons, as done in Otto's (2012) study. To classify different areas of study, the researcher used the NCAA Growth, Opportunities, and Learning Strategies (GOALS) areas of study from the question that asks student-athletes to identify the area of study where their major falls. There are twelve categories: I have not yet chosen a major area of study; Biological 
Sciences (Zoology, Physiology, etc.); Business (Accounting, Marketing, Personnel, etc.);

Communications (Journalism, Public Relations, etc.); Education (Elementary, Special, etc.);

Engineering, Computer/Information Sciences; Exercise, Sports, Kinesiology; Humanities and

Fine Arts (Music, Religion, English, etc.); Physical Sciences and Mathematics (Chemistry, etc.);

Professional Studies (Nursing, Occupational Therapy, etc.); Social Sciences (Psychology, History, Economics, etc.); Other Academic Field. The researcher added the classification of "unlisted" or "undecided" to the first category, "I have not yet chosen a major area of study."

The study included an analysis of the trends of student-athletes who pursued academic majors in particular areas of study to provide context for the phenomenon of clustering by academic major. Research Questions and Hypotheses

The following research questions were considered for this study: 1) What is the frequency of clustering by academic major at select HBCUs? 2) What is the most common academic major among student-athletes at select HBCUs? 3) What is the most common area of study among student-athletes at select HBCUs? The researcher hypothesized that clustering by academic major at HBCUs occurred, that sport management and physical education would be the most common academic majors among student-athletes at HBCUs, and that Exercise, Sports, and Kinesiology would be the most common area of study among student-athletes at HBCUs. Ad hoc analyses were conducted based upon findings from pilot research and previous research studies about clustering (Sanders \& Hildenbrand, 2010; Otto, 2012). The analyses investigated whether reports of clustering differed when clustering was reported within general areas of study rather than specific academic majors, whether clustering occurred more frequently within football or basketball programs, and whether data support the structural hypothesis of clustering (Sanders \& Hildenbrand, 2010). 


\section{Sampling/Recruitment}

The researcher collected data in January 2015. The NCAA Division I conference utilized in this study was the Mid-Eastern Athletic Conference (MEAC), comprised of thirteen HBCU institutions. The NCAA Division II conference utilized in this study was the Central Intercollegiate Athletic Association (CIAA), comprised of twelve institutions, eleven of which are HBCUs. Data from 9 of the 13 MEAC institutions and 9 of the 12 CIAA institutions for a total of 18 of the 25 institutions was available for the study. Sports Information Directors (SIDs) were contacted if the data was not available on the institution's athletic department website or elsewhere on the Internet. There were various reasons why data from the other HBCUs were not obtained: some institutions chose to exclude their student-athletes' academic major from the media guides, other institutions allowed their student-athletes to decide whether to include their academic major in the media guide, some institutions do not publish or print their media guides, and other institutions reported that they do not have the information at all. The researcher requested information regarding student-athlete majors at specific institutions from the NCAA, but learned that member institutions are expected to police themselves in academic matters.

The researcher analyzed media guides over a four-year window: 2009-2013. This window included football rosters for the following seasons: 2009, 2010, 2011, and 2012, and men's basketball rosters for the following seasons: 2009-2010, 2010-2011, 2011-2012, 20122013. The data contained 4174 football student-athletes and 922 basketball student-athletes. $49.2 \%$ of the student-athletes competed in the MEAC and $50.8 \%$ of the student-athletes competed in the CIAA. The researcher expanded the pilot research and included student-athletes from all academic years in school. The inclusion of student-athletes from all academic years in school was intended to reveal potential trends in student-athlete movement among academic 
majors. Assuming that data for each season of competition at the sample universities was available for the researcher, there were data for 144 seasons among the eighteen universities. However, the number of seasons decreased as it became apparent that data was not available for certain seasons at different universities.

Measures, Variables, and Procedure

The researcher categorized the student-athletes by their year in school and academic major. Student-athletes in their red-shirt years were categorized separately form student-athletes who did not take a red-shirt year. Student-athletes whose listed academic majors were actually a concentration of a broader major were listed under the broad major. For example, at one institution a student-athlete was listed as an accounting major. An investigation of the institution's website revealed that accounting is a concentration of the business administration major. Therefore, the student-athlete was listed as a business administration major. Additionally, analyses for each institution were conducted for each individual season by sport. In addition to each year of the season, each student-athlete's year in school, and each student-athlete's academic major, the researcher identified the specific department or college where each academic major is housed. For example, one institution may house their sport management academic major in the College of Business. In this example, College of Business was recorded along with the academic major of sport management.

Due to minor differences in the way that schools label similar academic majors (e.g. business management vs. management or criminal justice vs. justice studies), the researcher gave similar majors the same subheading. Student-athletes who completed double-majors were counted as two separate student-athletes. To assist in the classification of academic majors into their proper GOALS area of study, the researcher asked two other graduate students in sport and 
exercise psychology to assign each major to an area of study in an effort to eliminate potential bias from the researcher.

A codebook was created to input the data into Microsoft Excel spreadsheets. One of the issues with analyzing clustering by academic major across consecutive seasons is how to count the total number of student-athletes throughout the seasons. To mitigate that issue, all analyses of clustering were done in each individual season. The overall trends were computed by comparing each individual season's results.

IBM SPSS was used to compare and analyze statistics. To compare the data of the student-athletes' majors to non-athlete students' majors, previous researchers have used z-scores and chi-squared tests of independence (Case, Brown, \& Greer, 1987; Fountain \& Finley, 2009; Schneider, Ross, \& Fisher, 2010). This study did not use any measure of statistical analysis, instead used observed and analyzed frequency distributions to address the three research questions.

\section{Results}

The tables in Appendix A display the most popular academic majors and the rate in which student-athletes pursued them. The tables for football seasons include the top three academic majors and the tables for basketball seasons include the top two academic majors. In the tables, one asterisk denotes clustering and two asterisks denote extreme clustering. In seasons where the highest frequency of academic majors was undecided or unlisted, asterisks were not used to denote clustering.

Research Question 1: What is the frequency of clustering by academic major at select HBCUs?

For the eighteen schools analyzed in the study, there were data available for 117 of the possible 144 total seasons, 50 of the 68 possible football seasons and 67 of the possible 72 
basketball seasons. Clustering occurred in 5 of the 50 football seasons (10\%) and 30 of the 67 basketball seasons (45\%). The clustered academic major differed for each school and each sport team. For example, sport management was the clustered academic major at University 13 on the football and men's basketball teams; however, communications was the clustered academic major at University 10 on the men's basketball team while the football team had no seasons in which clustering occurred.

In the four years of football data made available for the nine institutions in the MEAC, clustering by academic major only occurred during one season and the clustering only occurred at one institution. There is the possibility that more clustering occurred; however, this cannot be confirmed because of the number of student-athletes whose academic major was undecided or unlisted in the media guide. Of the 2,012 pieces of data for football student-athletes in the MEAC over the four seasons, $40 \%$ of the student-athletes had undecided or unlisted academic majors (Table 1).

In the four years of football data made available for the nine institutions in the CIAA, clustering by academic major occurred four times. The clustering occurred during three seasons at one institution and occurred at two institutions. Of the 2,159 pieces of data for football student-athletes in the CIAA, $22 \%$ of the student-athletes had undecided or unlisted academic majors (Table 2).

In the four years of basketball data made available for the nine institutions in the MEAC, clustering by academic major occurred ten times. The clustering occurred at least once in each of the four seasons for analysis and occurred at four institutions. Of the 492 pieces of data for basketball student-athletes in the MEAC, 24\% of the student-athletes had undecided or unlisted academic majors (Table 3 ). 
In the four years of basketball data made available for the nine institutions in the CIAA, clustering by academic major occurred eighteen times. The clustering occurred at least once in each of the four seasons for analysis and occurred at five institutions. Of the 492 pieces of data for basketball student-athletes in the CIAA, $12 \%$ of the student-athletes had undecided or unlisted academic majors (Table 4).

However, in each of the 117 total seasons reviewed, the student-athletes on the football and men's basketball team pursued academic majors at a rate higher than the general student body. Data for the rates of student body enrollment by academic major were made available for 13 of the 18 schools. The rate at which the general student body pursued the most popular academic majors of student-athletes was smaller than those of student-athletes. In seasons where the majority of student-athletes had an undecided or unlisted major, there was no comparison to the general student body (Tables $5 \& 6$ ).

Research Question 2: What is the most common academic major among student-athletes at select HBCUs?

This question was addressed by analyzing the total number of student-athletes who pursued the academic major each year. The three most common academic majors for studentathletes at HBCUs over the four-year period were sport management (440), criminal justice (367), and business management (238). Sport management was a listed major at eleven of the eighteen universities. Seven of those universities are CIAA members. Criminal justice was a listed major at all eighteen universities. Business management was a listed major at thirteen of the eighteen universities. Six of those universities are CIAA members. A total of 1455 of the 5093 pieces of student-athlete data were unlisted or undecided academic majors, 920 of those from MEAC members and 535 of those from CIAA members (Table 7). 
The number of student-athletes who majored in sport management at MEAC universities steadily rose over the four year time period, while the number of student-athletes with the same major at CIAA universities rapidly increased over the four year time period. A similar trend occurred with student-athletes who majored in criminal justice at MEAC and CIAA universities. Regarding the numbers of student-athletes who pursued business management majors, MEAC universities showed a steady increase in the number of student-athletes while CIAA universities showed a rise and drop in the number of majors throughout the four year time period.

Research Question 3: What is the most common area of study among student-athletes at select HBCUs?

The most common areas of study among student-athletes at select HBCUs are Exercise, Sports, \& Kinesiology, Business, and Social Sciences (Table 8). The academic majors assigned to each of these NCAA GOALS Areas of Study can be found in Appendix C.

Ad Hoc Analyses

In addition to the research questions, the researcher performed ad hoc analyses to reveal more about the trends in clustering by academic major in this data set. The ad hoc analyses conducted made additional comparisons between the general student body and the studentathletes in the data set, tested the structural hypothesis of clustering (Sanders \& Hildenbrand, 2010), and addressed the challenges of accurately measuring clustering by academic major as compared to area of study (Otto, 2012).

Does the frequency of clustering increase when results are reported by area of study instead of academic major?

Otto (2012) indicated that reports of clustering by academic major increase when a researcher uses an area of study to record their data instead of an academic major. The researcher 
conducted this analysis and found that Otto's (2012) indication proved correct. Clustering by area of study occurred in 19 of the 50 football seasons (38\%) and 48 of the 67 basketball seasons $(72 \%)$. Also, clustering by area of study occurred at eight of the nine universities in this study in the MEAC and all nine of the universities in this study from the CIAA (Tables 10, 11, 12, \& 13). At what frequency do student-athletes pursue academic majors in different departments or colleges on their respective campus?

The structural hypothesis of clustering (Sanders \& Hildenbrand, 2010) states that one possible explanation for clustering by academic major among student-athletes is that studentathletes pursue academic majors that hold classes at times that fit best with their athletic schedules. An analysis of the frequency in which student-athletes pursued academic majors in different departments or colleges at each university revealed data that support the structural hypothesis. Furthermore, the fact that multiple clusters by academic major and area of study existed on teams also supports the structural hypothesis.

Is there a difference between the frequency at which clustering by academic major occurs in football and basketball programs?

There appeared to be a difference in the frequency at which clustering occurred among football and basketball teams. Clustering occurred at a larger number of institutions in basketball than in football and in a larger percentage of basketball seasons than football seasons. A chisquared test of independence was conducted to analyze the difference in the frequency of clustering. The chi-squared test of independence was significant with a moderate effect size. $\mathrm{X}^{2}$ $(1,117)=16.52, p<.001, \phi=.376$. Of all of the teams were clustering by academic major occurred, $86 \%$ of the teams were basketball teams. 


\section{Discussion}

This study examined clustering by academic major at HBCUs across two NCAA divisions, NCAA Division I and NCAA Division II. HBCUs have had their current relevance questioned and have been publicly shamed in media and non peer-reviewed sources as "academic wastelands" and "cheap and inferior institutions in comparison to traditional or mainstream higher education," (Brown II, 2013, p.4-5) but many of these universities are NCAA members and student-athletes that compete at these universities face similar challenges to those faced by student-athletes at all NCAA member institutions. The results show that clustering by academic major and area of study existed at institutions in both NCAA divisions. These findings are consistent with previous studies that examined clustering (Fountain \& Finley, 2009; Fountain \& Finley, 2011; Otto, 2012). In fact, the clustered majors at eight of the eleven ACC universities in Fountain and Finley's 2009 study fall under the most common areas of study among studentathletes at the universities in this study. Also, the clustered majors at four of the seven PAC-10 universities in Otto's 2012 study fall under the most common areas of study among studentathletes at the universities in this study. When the findings were separated by NCAA Division, they revealed minimal differences in the percentages of student-athletes in each area of study (Table 9). This difference can be explained by the availability of certain majors at each university.

Previous studies did not compare the frequencies of clustering in basketball and football programs. Despite the fact that there was a significant difference between the number of basketball and football teams where clustering occurred, the size of each team accounts for the differences in rates of clustering. Basketball teams carry anywhere between 8 and 18 players. On the other hand, football teams carry anywhere between 45 and 95 players. Therefore, the number 
of student-athletes pursuing the same academic major will account for a larger percentage on a basketball team than a football team.

The results of this study broaden the scope of investigations of clustering and raise the question of whether clustering is a natural by-product of intercollegiate sport participation. Former NCAA President Myles Brand has been documented saying, "Clustering itself is not inherently good or bad (Hollencamp, 2009)." Although research using media guides and rosters reveals that clustering by academic major occurs, there is the distinct possibility that the academic major that a student-athlete pursues is a result of their genuine personal interest and is not a result of other personal or systemic factors and influences.

Investigations of clustering and the processes behind decision-making for student-athletes are important because student-athletes are often at the root of blame for allegations of academic scandal at different institutions. Student-athletes are seen as solely responsible for making their own decisions about their academic major and eventual career path (Osborne, 2014). Yet, we do not know enough about the thought processes and analyses that lead student-athletes to make their decisions surrounding their academic major and first steps towards their careers. Often, a college student's academic major relates to their first job after college, and student-athletes that are clustered into academic majors that they did not choose can lead to first-job income gaps between student-athletes and the general student population (Sanders \& Hildenbrand, 2010).

There are several structural forces and influences that can impact a student-athlete's decision to pursue a major. The first and most important is that some departments and academic majors only offer classes in certain time slots or academic terms. If those time slots conflict with athletic obligations, there is a large chance that the student is either not permitted to take the class or chooses not to take the class. Second, student-athletes receive messages about which 
academic major to pursue from several sources, including but not limited to: coaches, athletic academic advisors, teammates, department academic advisors, faculty members, alumni, and family members. Third, student-athletes who transfer to an institution to compete are at the whim of their credits that transfer to that institution. Often, a student-athlete's decision to transfer to another institution for athletic reasons does not heavily weigh the academic consequences of the transfer. A student-athlete who decides to transfer to an institution to compete in his or her sport may consider the number of credits that transfer to the institution; however, the number of academic credits that transfer to the institution may not make or break the decision to switch universities. Finally, NCAA academic legislation places an emphasis on student-athletes maintaining academic standards in order to compete. If clustering by academic major or area of study is an intentional action by student-athletes to maintain their eligibility and cope with their athletic demands, further investigation into the impact of NCAA academic legislation and the overall structure of intercollegiate sports is warranted (Steeg, 2008; Elfman, 2009; Hollencamp, 2009; Dent, Sanserino, \& Werner, 2014).

There were several limitations in the study's method and scope that prevent the results of the research from being generalizable. First, the results of this study cannot be generalized because the scope is limited to HBCUs in two conferences. Furthermore, these institutions compete across different divisions. Second, the study's scope is only limited to student-athletes participating in football and men's basketball, which limits the scope to male student-athletes. Third, there are limitations to using published media guides as the primary source or data. There is the chance that the data published in the media guide did not officially come from the registrar's office or another academic division at the university. In those cases, the academic major listed in the media guide could be incorrect in one of a number of ways: the academic 
major listed is a concentration of the actual academic major, the academic major listed in the media guide is not offered by the university, or the student-athletes list the academic major differently than the university lists the major. Also, the print publications or online publications cannot account for a student-athlete's change in major. There is no guarantee that the publication will be updated if a student-athlete changes his major. Also, some athletic departments did not require student-athletes to publish their academic major, so there were many players at some institutions that did not have an academic major listed in the media guide. Rosters with significant numbers of unlisted majors altered the analysis and affected the accuracy of the reported results, not to mention the classification of a major as clustered or not. Furthermore, student-athletes who transfer to a university to play sports, follow a different timeline in declaring an academic major (Cooper \& Hawkins, 2014). Many of the student-athletes on the teams included in the study have a notable percentage of transfer student-athletes, primarily from junior colleges or community colleges. These student-athletes face different rules related to the deadline to declare an academic major. Therefore, community college and junior college transfer student-athletes who are listed as sophomores or juniors in the media guide may have an unlisted or undecided major because they are not required to have one unlike sophomores and juniors who started their freshman year at the four-year institution.

Other limitations exist based upon the number of seasons the researcher examined. Although this study's scope extended across four years, the NCAA allows student-athletes a maximum of six years to graduate. In those cases, student-athletes may participate in sport for up to four years and then enroll as non-athlete students to complete the requirements for their degree, which could include a change in academic major. Furthermore, the researcher did not have access to information about student-athlete entering skills such as standardized test scores, high 
school grade point average, or other information that could influence one's academic major. Undoubtedly, a student's entering skills as well as the grades that they earn during their initial years in college can influence the major that he or she pursues (Sanders \& Hildenbrand, 2010).

The gaps in research about clustering by academic major are very clear. It is evident that clustering has happened, is happening, and probably will continue to happen in men's basketball and football. However, none of the research has sought to obtain qualitative data from students about the decision to major in a field that has so many of their teammates represented. Authors of different articles have attempted to explain clustering but none of these explanations have been empirically researched. These explanations state that clustering happens for one or some combination of the following reasons: to avoid faculty members who harbor resentment for student-athletes, to flock towards faculty members who are more flexible with student-athletes, to be close to teammates for social and academic support, and to choose a major that allows the student-athlete more time to dedicate to sport.

In addition to the aforementioned suggestions, there are several other directions that would yield great amounts of information about academic clustering and the culture of revenuegenerating sports as a whole. Although previously studied, one important future direction of research should examine the number of student-athletes who move out of non-clustered majors into clustered major after their first or second year of competition. A completely unstudied area future research on clustering is the rates of clustering in non-revenue generating sports, women's sports, or revenue generating sports in other NCAA divisions or other college sports organizations. Another direction future research can take is to investigate coaches' attitudes and knowledge about clustering in their programs. Research to investigate how student-athletes who have completed college have used information learned through their major in their career 
pursuits or life after college would be an informative future direction as well. Also, research that investigates athletic academic advisors about their attitudes and beliefs about clustering or advising a group of student-athletes to pursue the same major or area of study.

Revenue generating sports are not limited to NCAA Division I. Men's basketball and football are revenue generating sports in NCAA Divisions II and III despite the fact that these sports do not accrue as much revenue as the "big-time" Division I programs. By investigating clustering across NCAA Divisions and in non-revenue sports, researchers and university employees can gain perspective about the clustering that exists in revenue-generating sports. There have been no peer-reviewed publications regarding clustering from the student-athlete perspective. All peer-reviewed publications have examined media guides, but have not interviewed student-athletes, athletic academic advisors, coaches, faculty members, or anyone else who plays an important role in a student-athlete's decision to pursue a certain academic major. Without this data, one can inaccurately attribute the source of this issue, which can lead to new legislation or policy implementation that does not provide any more support for studentathletes. Research surrounding the aforementioned topics can reveal more about the studentathlete academic experience as a whole and provide a much-needed context for the recent academic scandals surrounding student-athletes in revenue generating sports. 


\section{References}

Berkowitz, S., Upton, J., \& Schnaars, C. (2013, June 11). It's academic: Coaches, ADs, collect APR bonuses. USA Today. Retrieved from http://www.usatoday.com/story/sports/college/2013/06/11/ncaa-apr-bonuses-coachathletics-director/2412095/

Bettez, S. C., \& Suggs, V. L. (2012). Centering the educational and social significance of hbcus: A focus on the educational journeys and thoughts of african american scholars. Urban Review, 44, 303-310. Doi: 10.1007/s11256-012-0201-x

Cantey, N. I., Bland, R., Mack, L. R., \& Joy-Davis, D. (2013). Historically black colleges and universities: Sustaining a culture of excellence in the twenty-first century. Journal of African American Studies, 17, 142-153. Doi: 10.1007/s12111-011-9191-0

Case, B., Greer, S., \& Brown, J. (1987). Academic clustering in athletics: Myth or reality? Arena Review, 11(2), pp. 48-56.

Cooper, J. N., Hawkins, B. (2012). A place of opportunity: Black male student athletes' experiences at a historically black university. Journal of Intercollegiate Sport, 5, 170-188.

Cooper, J. N., Hawkins, B. (2014). The transfer effect: A critical race theory examination of black male transfer student athletes' experiences. Journal of Intercollegiate Sport, 7, 80104.

Coupet, J. (2013). Historically black colleges and universities and resource dependence: A Chow test of production functions. Journal of Higher Education Policy and Management, 35(4), 355-369. Doi: 10.1080/1360080X.2013.812054

Davenport, J. (1985). From crew to commercialism. In D. Chu, J.O. Seagrave, \& B.J. Becker 
(Editors), Sport in higher education (5-15). Champaign, IL. Human Kinetics Publishers, Inc.

Fountain, J. J., and Finley, P. S. (2009). Academic majors of upperclassmen football players in the atlantic coast conference: An analysis of academic clustering comparing white and minority players. Journal of Issues in Intercollegiate Athletics, 2, 1-13.

Fountain, J. J., and Finley, P. S. (2011). Academic clustering: A longitudinal analysis of a division I football program. Journal of Issues in Intercollegiate Athletics, 4, 24-41.

Gasman, M. (2011). The changing face of historically black colleges and universities. University of Pennsylvania Graduate School of Education. Retrieved from http://www.gse.upenn.edu/changing_face_hbcus

Hollencamp, K. (2009, February 23). NCAA academic ratings may force students to choose between dreams. Medill Reports. Retrieved from http://news.medill.northwestern.edu/Chicago/news.aspx?id=118185\&print=1

Johnson, M. N. (2013). Financial and related issues among historically black colleges and universities. Journal of Intercollegiate Sport, 6, 65-75.

Kim, M.M., \& Conrad, C.F. (2006). The impact of historically black colleges and universities on the academic success of african-american students. Research in Higher Education, 47(4), 399-427. Doi: 10.1007/s11162-005-9001-4

Lanter, J. R., \& Hawkins, B. J. (2013). The economic model of intercollegiate athletics and its effects on the college athlete educational experience. Journal of Intercollegiate Sport, 6, 86-95.

Melendez, M. C. (2010). Psychosocial influences on college adjustment in division 1 student- 
athletes: The role of athletic identity. Journal of College Student Retention, 11(3), 345361.

National Center for Education Statistics, Institute of Education Sciences, U.S. Department of Education. Integrated Postsecondary Education Data System (IPEDS), Spring 2010, Graduation Rates component.

National Center for Education Statistics, Institute of Education Sciences, U.S. Department of Education. Integrated Postsecondary Education Data System (IPEDS), Fall 2011, HBCU Enrollment Rates component.

National Collegiate Athletic Association (n.d.). NCAA Students Facebook Page. Retrieved from https://www.youtube.com/watch?v=CXeDUFTaU1Y\&spfreload=10

Nichols, J. C. (2004). Unique characteristics, leadership styles, and management of historically black colleges and universities. Innovative Higher Education, 28(3), 219-229. Doi: 10.1023/B:IHIE.0000015109.49156.fb

Nyquist, E.B. (1985). The immorality of big-power intercollegiate athletics. In D. Chu, J.O. Seagrave, \& B.J. Becker (Editors), Sport in higher education (101-114). Champaign, IL. Human Kinetics Publishers, Inc.

Osborne, B. (2014). The myth of the exploited student-athlete. Journal of Intercollegiate Sport, 7 , 143-152.

Otto, K. (2012). Demonstrating the importance of accuracy in reporting results of academic clustering. Journal for the Study of Sports and Athletes in Education, 3, 293-310. DOI: http://dx.doi.org/10.1179/ssa.2012.6.3.293

PayScale (2015). College salary report 2014-15. Retrieved from 
http://www.payscale.com/college-salary-report/majors-that-pay-youback/bachelors\#explanatory

Purdy, D. A., Eitzen, D. S. \& Hufnagel, R. (1982). Are athletes also students? The educational attainment of college athletes. Social Problems, 29, 439-448. DOI: 10.2307/800032

Renick, J. (1974). The use and misuse of college athletics. The Journal of Higher Education, 45(7), 545-552. DOI: 10.2307/1980793

Sack, A. (1986). Jan Kemp wins one for integrity. Journal of Sport and Social Issues, 10(1), 3-5. Doi: $10.1177 / 019372358601000101$

Sanders, J. P., \& Hildenbrand, K. (2010). Major concerns? A longitudinal analysis of studentathletes' academic majors in comparative perspective. Journal of Intercollegiate Sport, 3, 213-233.

Schneider, R. G., Ross, S. R., \& Fisher, M. (2010). Academic clustering and major selection of intercollegiate student-athletes. College Student Journal, 44(1), 64-70.

Sellers, R. M., Chavous, T. M., \& Brown, T, N. (2002). Uneven playing field: The impact of structural barriers on the initial eligibility of African American student-athletes. Paradoxes of Youth and Sport, 173-186.

Sharp, L. A., \& Sheilley, H. K. (2008). The 28merica28ion's obligations to athletes. New Directions for Higher Education, 142, 103-113.

Shropshire, K. L. (2013). Complexity and memory: Financial related issues among hbcus. Journal of Intercollegiate Sport, 6, 76-78.

Sojka, G.S. (1985). The evolution of the student-athlete in America: From the divinity to the divine. In D. Chu, J.O. Seagrave, \& B.J. Becker (Editors), Sport in higher education (1733). Champaign, IL. Human Kinetics Publishers, Inc. 
Steeg, J. (2008). UNLV athletes question degrees in university studies. USA Today. Retrieved from http://www.usatoday.com/sports/college/2008-11-19-unlv-university-studiesdegree N.htm

Stuart, R. (2012). HBCUs could be hit hard by new NCAA rules. Diverse Education. Retrieved from http://diverseeducation.com/article/17098/

Suggs, W. (2003). Jock majors: Many colleges allow football players to take the easy way out. The Chronicle of Higher Education, 49(17), 33. Retrieved from http://chronicle.com/article/Jock-Majors/32843 


\section{Appendix A}

Table 1

Mid-Eastern Athletic Conference Football Academic Majors

\begin{tabular}{|c|c|c|c|c|}
\hline & 2009 & 2010 & 2011 & 2012 \\
\hline University 1 & N/A & N/A & $\begin{array}{l}\text { Unlisted/Undecided (44\%) } \\
\text { Management }(9 \%)\end{array}$ & $\begin{array}{l}\text { Unlisted/Undecided }(45 \%) \\
\text { Movement Science }(16 \%) \\
\text { Criminal Justice }(8 \%)\end{array}$ \\
\hline University 2 & $\begin{array}{c}\text { Unlisted/Undecided } \\
(47 \%) \\
\text { Sport Management } \\
(12 \%) \\
\text { Psychology }(9 \%)\end{array}$ & $\begin{array}{c}\text { Unlisted/Undecided } \\
\qquad(27 \%) \\
\text { Psychology }(21 \%) \\
\text { Sport Management } \\
(12 \%)\end{array}$ & $\begin{array}{c}\text { Unlisted/Undecided (27\%) } \\
\text { Psychology (11\%) } \\
\text { Criminal Justice (8\%) }\end{array}$ & $\begin{array}{c}\text { Unlisted/Undecided (21\%) } \\
\text { Sport Management (14\%) } \\
\text { Psychology }(9 \%)\end{array}$ \\
\hline University 3 & No Football Program & No Football Program & No Football Program & No Football Program \\
\hline University 4 & N/A & $\begin{array}{l}\text { Unlisted/Undecided } \\
(74 \%)\end{array}$ & Unlisted/Undecided (70\%) & Unlisted/Undecided (74\%) \\
\hline University 5 & $\begin{array}{c}\text { Business (13\%) } \\
\text { Interdisciplinary Studies } \\
(9 \%) \\
\text { Exercise Science }(9 \%)\end{array}$ & $\begin{array}{c}\text { Unlisted/Undecided } \\
(21 \%) \\
\text { Business } \\
\text { Management }(14 \%) \\
\text { Interdisciplinary } \\
\text { Studies }(8 \%)\end{array}$ & $\begin{array}{c}\text { Unlisted/Undecided (29\%) } \\
\text { Business Management } \\
(15 \%) \\
\text { Interdisciplinary Studies } \\
(9 \%)\end{array}$ & $\begin{array}{c}\text { Unlisted/Undecided (15\%) } \\
\text { Sociology (14\%) } \\
\text { Interdisciplinary Studies } \\
(9 \%)\end{array}$ \\
\hline University 6 & N/A & $\begin{array}{c}\text { Unlisted/Undecided } \\
(84 \%)\end{array}$ & Unlisted/Undecided (58\%) & Unlisted/Undecided (50\%) \\
\hline University 7 & $\begin{array}{c}\text { Unlisted/Undecided } \\
(20 \%) \\
\text { Criminal Justice }(16 \%) \\
\text { Business Management } \\
(14 \%)\end{array}$ & $\begin{array}{c}\text { Criminal Justice } \\
(25 \%)^{*} \\
\text { Sport Management } \\
(15 \%) \\
\text { Business } \\
\text { Management }(10 \%)\end{array}$ & $\begin{array}{c}\text { Sport Management }(15 \%) \\
\text { Unlisted/Undecided (13\%) } \\
\text { Criminal Justice }(12 \%)\end{array}$ & $\begin{array}{c}\text { Unlisted/Undecided (18\%) } \\
\text { Criminal Justice }(10 \%) \\
\text { Sport Management }(9 \%)\end{array}$ \\
\hline University 8 & $\begin{array}{l}\text { Unlisted/Undecided } \\
(83 \%)\end{array}$ & N/A & N/A & N/A \\
\hline University 9 & N/A & $\begin{array}{c}\text { Unlisted/Undecided } \\
(37 \%) \\
\text { Physical Education } \\
(15 \%) \\
\text { Criminal Justice } \\
(8 \%)\end{array}$ & $\begin{array}{l}\text { Unlisted/Undecided (44\%) } \\
\text { Physical Education (19\%) } \\
\text { Criminal Justice (8\%) }\end{array}$ & $\begin{array}{c}\text { Unlisted/Undecided (29\%) } \\
\text { Physical Education (13\%) } \\
\text { Criminal Justice (10\%) }\end{array}$ \\
\hline
\end{tabular}


Table 2

Central Intercollegiate Athletic Association Football

\begin{tabular}{|c|c|c|c|c|}
\hline & 2009 & 2010 & 2011 & 2012 \\
\hline University 10 & $\begin{array}{c}\text { Business } \\
\text { Administration (9\%) } \\
\text { Business } \\
\text { Management (8\%) } \\
\text { Sport Management } \\
\text { (8\%) }\end{array}$ & $\begin{array}{c}\text { Sport Management } \\
(15 \%) \\
\text { Communications }(10 \%) \\
\text { Computer Technology } \\
(9 \%) \\
\text { Business Management } \\
(9 \%)\end{array}$ & $\begin{array}{c}\text { Sport Management }(15 \%) \\
\text { Criminal Justice }(10 \%) \\
\text { Accounting }(9 \%)\end{array}$ & $\begin{array}{c}\text { Sport Management (18\%) } \\
\text { Business Management } \\
(10 \%) \\
\text { Computer Technology } \\
(10 \%)\end{array}$ \\
\hline University 11 & $\begin{array}{c}\text { Physical Education } \\
(19 \%) \\
\text { Criminal Justice } \\
(16 \%) \\
\text { Sport Management } \\
(16 \%)\end{array}$ & $\begin{array}{c}\text { Physical Education } \\
(21 \%) \\
\text { Criminal Justice (14\%) } \\
\text { Sport Management } \\
(14 \%)\end{array}$ & $\begin{array}{l}\text { Physical Education }(15 \%) \\
\text { Criminal Justice }(13 \%) \\
\text { Sport Management }(13 \%)\end{array}$ & $\begin{array}{c}\text { Criminal Justice }(13 \%) \\
\text { Physical Education }(11 \%) \\
\text { Sport Management }(10 \%)\end{array}$ \\
\hline University 12 & N/A & $\begin{array}{c}\text { Unlisted/Undecided } \\
(38 \%) \\
\text { Criminal Justice (16\%) } \\
\text { Physical Education } \\
(14 \%)\end{array}$ & N/A & $\begin{array}{l}\text { Unlisted/Undecided (28\%) } \\
\text { Physical Education }(16 \%) \\
\text { Criminal Justice }(15 \%)\end{array}$ \\
\hline University 13 & $\begin{array}{c}\text { Unlisted/Undecided } \\
(31 \%) \\
\text { Sport Management } \\
(22 \%) \\
\text { Business } \\
\text { Administration } \\
(14 \%)\end{array}$ & $\begin{array}{c}\text { Sport Management } \\
(35 \%)^{*} \\
\text { Business Administration } \\
(16 \%) \\
\text { Computer Engineering } \\
(8 \%)\end{array}$ & $\begin{array}{c}\text { Sport Management } \\
(27 \%)^{*} \\
\text { Business Administration } \\
(13 \%) \\
\text { Computer Engineering } \\
(8 \%)\end{array}$ & $\begin{array}{c}\text { Sport Management }(25 \%)^{*} \\
\text { Unlisted/Undecided }(16 \%) \\
\text { Business Administration } \\
(9 \%)\end{array}$ \\
\hline University 14 & N/A & $\begin{array}{c}\text { Unlisted/Undecided } \\
(52 \%) \\
\text { Criminal Justice (8\%) } \\
\text { Computer Science (7\%) } \\
\text { Unlisted/Undecided }\end{array}$ & $\begin{array}{c}\text { Unlisted/Undecided } \\
(64 \%) \\
\text { Criminal Justice }(10 \%) \\
\text { Health Science }(6 \%)\end{array}$ & $\begin{array}{c}\text { Unlisted/Undecided (27\%) } \\
\text { Criminal Justice }(17 \%) \\
\text { Health Science }(11 \%)\end{array}$ \\
\hline University 15 & N/A & $\begin{array}{c}(16 \%) \\
\text { Human Performance \& } \\
\text { Wellness }(13 \%) \\
\text { Sport Management } \\
(12 \%)\end{array}$ & $\begin{array}{c}\text { General Studies }(27 \%)^{*} \\
\text { Sport Management }(13 \%) \\
\text { Business Administration } \\
(12 \%)\end{array}$ & $\begin{array}{c}\text { Sport Management }(18 \%) \\
\text { Unlisted/Undecided }(9 \%) \\
\text { Human Performance \& } \\
\text { Wellness }(9 \%)\end{array}$ \\
\hline University 16 & N/A & N/A & N/A & $\begin{array}{c}\text { Sport Management (15\%) } \\
\text { Criminal Justice (11\%) } \\
\text { Mass Communications } \\
(8 \%)\end{array}$ \\
\hline University 17 & N/A & N/A & N/A & $\begin{array}{c}\text { Criminal Justice }(35 \%)^{*} \\
\text { Computer Information } \\
\text { Systems }(10 \%) \\
\text { Entrepreneurship }(9 \%)\end{array}$ \\
\hline University 18 & $\begin{array}{c}\text { Unlisted/Undecided } \\
(27 \%) \\
\text { Accounting }(8 \%) \\
\text { Sport Management } \\
(7 \%)\end{array}$ & $\begin{array}{c}\text { Unlisted/Undecided } \\
(59 \%) \\
\text { Accounting }(4 \%) \\
\text { Computer Science }(4 \%)\end{array}$ & $\begin{array}{c}\text { Unlisted/Undecided } \\
(51 \%) \\
\text { Computer Science }(6 \%) \\
\text { Accounting }(5 \%)\end{array}$ & $\begin{array}{c}\text { Unlisted/Undecided (81\%) } \\
\text { Computer Science }(4 \%) \\
\text { Accounting }(3 \%)\end{array}$ \\
\hline
\end{tabular}


Table 3

Mid-Eastern Athletic Conference Basketball

\begin{tabular}{|c|c|c|c|c|}
\hline University 1 & $\begin{array}{c}\mathbf{2 0 0 9 - 2 0 1 0} \\
\text { Unlisted/Undecided } \\
(50 \%)\end{array}$ & $\begin{array}{c}\mathbf{2 0 1 0 - 2 0 1 1} \\
\text { Unlisted/Undecided } \\
(50 \%)\end{array}$ & $\begin{array}{c}\mathbf{2 0 1 1 - 2 0 1 2} \\
\text { Sport Management (33\%)* } \\
\text { Mass Communications } \\
(25 \%)\end{array}$ & $\begin{array}{c}\text { 2012-2013 } \\
\text { Sport Management }(33 \%)^{*} \\
\text { Mass Communications } \\
(20 \%)\end{array}$ \\
\hline University 2 & $\begin{array}{c}\text { Unlisted/Undecided } \\
(43 \%) \\
\text { Sport Management } \\
(29 \%)^{*}\end{array}$ & $\begin{array}{c}\text { Sport Management } \\
(31 \%)^{*} \\
\text { Recreation }(13 \%)\end{array}$ & $\begin{array}{c}\text { Sport Management (33\%)* } \\
\text { Recreation }(13 \%)\end{array}$ & $\begin{array}{l}\text { Sport Management }(29 \%)^{*} \\
\text { Unlisted/Undecided }(23 \%)\end{array}$ \\
\hline University 3 & $\begin{array}{c}\text { Accounting }(23 \%) \\
\text { Sociology }(23 \%)\end{array}$ & $\begin{array}{l}\text { Accounting (21\%) } \\
\text { General Studies (14\%) }\end{array}$ & $\begin{array}{l}\text { Unlisted/Undecided (38\%) } \\
\text { General Studies (15\%) }\end{array}$ & $\begin{array}{l}\text { General Studies (21\%) } \\
\text { English (14\%) }\end{array}$ \\
\hline University 4 & $\begin{array}{l}\text { Unlisted/Undecided } \\
(67 \%)\end{array}$ & $\begin{array}{c}\text { Unlisted/Undecided } \\
(60 \%)\end{array}$ & Unlisted/Undecided (43\%) & Unlisted/Undecided (54\%) \\
\hline University 5 & $\begin{array}{c}\text { Interdisciplinary } \\
\text { Studies }(15 \%) \\
\text { Mass } \\
\text { Communications } \\
(15 \%)\end{array}$ & $\begin{array}{c}\text { Physical Education (13\%) } \\
\text { Sociology }(13 \%)\end{array}$ & $\begin{array}{c}\text { Mass Communications } \\
(16 \%) \\
\text { Accounting }(11 \%)\end{array}$ & $\begin{array}{c}\text { Accounting }(20 \%) \\
\text { Interdisciplinary Studies } \\
(13 \%)\end{array}$ \\
\hline University 6 & $\begin{array}{c}\text { Unlisted/Undecided } \\
(27 \%) \\
\text { Sport Science \& } \\
\text { Fitness Management } \\
(13 \%)\end{array}$ & $\begin{array}{l}\text { Sport Science \& Fitness } \\
\text { Management }(18 \%) \\
\text { Graphic Communications } \\
\text { Systems }(12 \%)\end{array}$ & $\begin{array}{l}\text { Unlisted/Undecided (21\%) } \\
\text { Sport Science \& Fitness } \\
\text { Management (14\%) }\end{array}$ & $\begin{array}{l}\text { Sport Science \& Fitness } \\
\text { Management }(20 \%) \\
\text { Criminal Justice }(13 \%)\end{array}$ \\
\hline University 7 & $\begin{array}{c}\text { Sociology }(21 \%) \\
\text { Mass } \\
\text { Communications } \\
(14 \%)\end{array}$ & $\begin{array}{c}\text { Sociology }(26 \%)^{*} \\
\text { Unlisted/Undecided } \\
(21 \%)\end{array}$ & $\begin{array}{c}\text { Unlisted/Undecided (20\%) } \\
\text { Sociology }(13 \%)\end{array}$ & $\begin{array}{c}\text { Psychology }(26 \%)^{*} \\
\text { Sport Management }(20 \%)\end{array}$ \\
\hline University 8 & $\begin{array}{c}\text { Unlisted/Undecided } \\
(18 \%) \\
\text { Mathematics (13\%) }\end{array}$ & $\begin{array}{c}\text { Unlisted/Undecided } \\
(25 \%) \\
\text { Mass Communications } \\
(19 \%)\end{array}$ & $\begin{array}{c}\text { Unlisted/Undecided (20\%) } \\
\text { Biology (13\%) }\end{array}$ & $\begin{array}{c}\text { Unlisted/Undecided (31\%) } \\
\text { Mass Communications } \\
(23 \%)\end{array}$ \\
\hline University 9 & N/A & $\begin{array}{c}\text { Business Management } \\
(25 \%)^{*} \\
\text { Unlisted/Undecided } \\
(25 \%)\end{array}$ & $\begin{array}{c}\text { Physical Education (25\%)* } \\
\text { Sociology }(13 \%)\end{array}$ & N/A \\
\hline
\end{tabular}


Table 4

Central Intercollegiate Athletic Association Basketball

2009-2010

University

10

Communications $(25 \%)$ *

Sport Management (17\%)

University Sport Management (31\%)*

11

Engineering (13\%)

\section{University}

12

N/A

Sport Management (29\%)*

University

13 Computer Information Systems $(13 \%)$

University

14

$$
\text { Unlisted/Undecided (21\%) }
$$

Information Technology (14\%)

University

15

Human Performance \&
Wellness $(25 \%)^{*}$
Sport Management (13\%)

University

16

Mass Communications (25\%)*

Business Management (17\%)

University Unlisted/Undecided (33\%)

$17 \quad$ Marketing (13\%)

University

18

Unlisted/Undecided (28\%)

Criminal Justice (17\%)
2010-2011

Communications $(29 \%)^{*}$

Business (14\%)

N/A

$\mathrm{N} / \mathrm{A}$

Business

Administration

(20\%)

Sociology (13\%)

Sport Management $(50 \%) * *$

Unlisted/Undecided (33\%)

Business

Management (21\%)

Health Science

(14\%)

Sport Management $(32 \%)^{*}$

Human

Performance \&

Wellness (19\%)

Business

Management

$(31 \%)^{*}$

Sport Management (23\%)

N/A

Unlisted/Undecided $(40 \%)$

Criminal Justice $(20 \%)$
Communications $(50 \%) * *$ Business (14\%)

Communications $(42 \%) * *$

Sport Management (21\%)

Business Administration $(35 \%) *$

Physical Education (29\%)*

Unlisted/Undecided (26\%)

Business Administration

(13\%)

Sport Management $(67 \%) * *$

Unlisted/Undecided (17\%)

Biology (13\%)

Mass Communications

$$
(13 \%)
$$

Sport Management $(33 \%)^{*}$

Computer Information Systems (11\%)

Sport Management $(47 \%)^{* *}$

Business Management $(33 \%)^{*}$
N/A

N/A

Unlisted/Undecided (21\%) Biology (21\%)

Sport Management $(69 \%) * *$

Criminal Justice (6\%)

Biology (14\%)

Business (14\%)

Sport Management (36\%)* Business Administration (14\%)

Sport Management (33\%)* Mass Communications $(13 \%)$

N/A Unlisted/Undecided (47\%) Sport Management (12\%) 
Table 5

Mid-Eastern Athletic Conference General Student Population

\begin{tabular}{|c|c|c|c|c|}
\hline University 1 & 2009-2010 & 2010-2011 & $\begin{array}{c}\mathbf{2 0 1 1 - 2 0 1 2} \\
\text { Mass Communications } \\
(7 \%) \\
\text { Management }(3 \%) \\
\text { Sport Management }(.5 \%)\end{array}$ & $\begin{array}{c}\text { 2012-2013 } \\
\text { Mass Communications } \\
(7 \%) \\
\text { Movement Science }(6 \%) \\
\text { Sport Management }(.25 \%)\end{array}$ \\
\hline University 3 & $\begin{array}{c}\text { Sociology }(7 \%) \\
\text { Exercise Science } \\
(4 \%) \\
\text { Accounting }(3 \%)\end{array}$ & Accounting (3\%) & General Studies (5\%) & General Studies (4\%) \\
\hline University 5 & $\begin{array}{c}\text { Interdisciplinary } \\
\text { Studies (6\%) } \\
\text { Mass } \\
\text { Communications } \\
(5 \%) \\
\text { Physical Education } \\
\text { (5\%) } \\
\text { Exercise Science } \\
(5 \%)\end{array}$ & $\begin{array}{c}\text { Business }(11 \%) \\
\text { Business Management } \\
(11 \%) \\
\text { Interdisciplinary Studies } \\
(6 \%) \\
\text { Sociology }(6 \%) \\
\text { Physical Education } \\
(5 \%)\end{array}$ & $\begin{array}{c}\text { Business }(10 \%) \\
\text { Interdisciplinary Studies } \\
(6 \%) \\
\text { Mass Communications } \\
(5 \%) \\
\text { Accounting }(3 \%)\end{array}$ & $\begin{array}{c}\text { Sociology }(7 \%) \\
\text { Interdisciplinary Studies } \\
(6 \%) \\
\text { Sociology }(6 \%)\end{array}$ \\
\hline University 6 & $\begin{array}{c}\text { Sport Science \& } \\
\text { Fitness Management } \\
(4 \%)\end{array}$ & $\begin{array}{c}\text { Sport Science \& Fitness } \\
\text { Management }(4 \%) \\
\text { Criminal Justice }(3 \%) \\
\text { Management }(.6 \%)\end{array}$ & $\begin{array}{l}\text { Sport Science \& Fitness } \\
\text { Management }(4 \%) \\
\text { Management }(1 \%)\end{array}$ & $\begin{array}{l}\text { Sport Science \& Fitness } \\
\text { Management }(5 \%) \\
\text { Criminal Justice }(4 \%) \\
\text { Management }(1 \%)\end{array}$ \\
\hline University 7 & $\begin{array}{c}\text { Business } \\
\text { Management (11\%) } \\
\text { Criminal Justice } \\
(8 \%) \\
\text { Mass } \\
\text { Communications } \\
(3 \%) \\
\text { Sociology }(1 \%)\end{array}$ & $\begin{array}{c}\text { Business Management } \\
(11 \%) \\
\text { Criminal Justice }(9 \%) \\
\text { Mass Communications } \\
(3 \%) \\
\text { Sociology }(1 \%)\end{array}$ & $\begin{array}{c}\text { Criminal Justice }(10 \%) \\
\text { Sociology }(1 \%)\end{array}$ & $\begin{array}{c}\text { Criminal Justice }(10 \%) \\
\text { Psychology }(6 \%) \\
\text { Mass Communications } \\
(4 \%)\end{array}$ \\
\hline University 8 & $\begin{array}{c}\text { Computer } \\
\text { Engineering (4\%) } \\
\text { Civil Engineering } \\
\quad(3 \%) \\
\text { Mathematics }(2 \%)\end{array}$ & $\begin{array}{c}\text { Biology }(10 \%) \\
\text { Business Management } \\
(9 \%) \\
\text { Communications }(8 \%) \\
\text { Chemical Engineering } \\
\text { (4\%) }\end{array}$ & $\begin{array}{c}\text { Biology (11\%) } \\
\text { Business Management } \\
(8 \%) \\
\text { Communications (8\%) } \\
\text { Accounting (5\%) } \\
\text { Computer Engineering } \\
(4 \%)\end{array}$ & $\begin{array}{c}\text { Communications (8\%) } \\
\text { Business Management } \\
\text { (7\%) } \\
\text { Computer Engineering } \\
(3 \%)\end{array}$ \\
\hline University 9 & N/A & $\begin{array}{c}\text { Data for Business } \\
\text { Management majors not } \\
\text { available. }\end{array}$ & $\begin{array}{c}\text { Sociology }(9 \%) \\
\text { Data for Physical } \\
\text { Education majors not } \\
\text { available. }\end{array}$ & \\
\hline
\end{tabular}


Table 6

Central Intercollegiate Athletic Association General Student Population

\begin{tabular}{|c|c|c|c|c|}
\hline University 10 & 2009-2010 & $\begin{array}{c}\text { 2010-2011 } \\
\text { Communications }(9 \%) \\
\text { Sport Management }(1 \%)\end{array}$ & $\begin{array}{c}\text { 2011-2012 } \\
\text { Communications }(9 \%) \\
\text { Sport Management }(2 \%)\end{array}$ & $\begin{array}{c}\text { 2012-2013 } \\
\text { Business Administration } \\
(19 \%) \\
\text { Communications }(9 \%) \\
\text { Sport Management }(2 \%)\end{array}$ \\
\hline University 11 & $\begin{array}{c}\text { Criminal Justice (8\%) } \\
\text { Physical Education } \\
(6 \%) \\
\text { Data for sport } \\
\text { management not } \\
\text { available. }\end{array}$ & $\begin{array}{c}\text { Criminal Justice (8\%) } \\
\text { Physical Education }(6 \%) \\
\text { Data for sport } \\
\text { management not } \\
\text { available. }\end{array}$ & $\begin{array}{c}\text { Criminal Justice }(9 \%) \\
\text { Physical Education }(6 \%) \\
\text { Data for sport } \\
\text { management not available. }\end{array}$ & $\begin{array}{c}\text { Criminal Justice }(9 \%) \\
\text { Physical Education }(6 \%) \\
\text { Data for sport management } \\
\text { not available. }\end{array}$ \\
\hline University 12 & N/A & $\begin{array}{c}\text { Business Administration } \\
\text { (11\%) } \\
\text { Criminal Justice (9\%) }\end{array}$ & $\begin{array}{c}\text { Business Administration } \\
\qquad(11 \%)\end{array}$ & $\begin{array}{c}\text { Criminal Justice (7\%) } \\
\text { Biology (4\%) }\end{array}$ \\
\hline University 14 & $\begin{array}{c}\text { Business } \\
\text { Management }(10 \%) \\
\text { Health Science }(1 \%) \\
\text { Information } \\
\text { Technology }(1 \%) \\
\text { Sociology }(1 \%)\end{array}$ & $\begin{array}{c}\text { Business Management } \\
(10 \%) \\
\text { Criminal Justice }(9 \%) \\
\text { Computer Science }(3 \%)\end{array}$ & $\begin{array}{c}\text { Criminal Justice }(11 \%) \\
\text { Business }(10 \%) \\
\text { Biology }(9 \%) \\
\text { Communications }(5 \%) \\
\text { Health Science }(7 \%)\end{array}$ & $\begin{array}{c}\text { Biology (14\%) } \\
\text { Criminal Justice (12\%) } \\
\text { Business (9\%) } \\
\text { Communications (5\%) } \\
\text { Health Science }(5 \%)\end{array}$ \\
\hline University 16 & $\begin{array}{c}\text { Mass } \\
\text { Communications } \\
(8 \%)\end{array}$ & $\begin{array}{c}\text { Mass Communications } \\
(8 \%) \\
\text { Business Management } \\
(7 \%) \\
\text { Data for sport } \\
\text { management not } \\
\text { available. }\end{array}$ & $\begin{array}{c}\text { Business Management } \\
\text { ( } 7 \%) \\
\text { Data for sport } \\
\text { management not available. }\end{array}$ & $\begin{array}{c}\text { Mass Communications } \\
(7 \%) \\
\text { Business Management } \\
(7 \%) \\
\text { Data for sport management } \\
\text { not available. }\end{array}$ \\
\hline University 18 & $\begin{array}{c}\text { Business } \\
\text { Administration (6\%) } \\
\text { Psychology (6\%) } \\
\text { Exercise Science } \\
(5 \%) \\
\text { Criminal Justice (3\%) } \\
\text { Accounting }(2 \%) \\
\text { Sport Management } \\
(2 \%)\end{array}$ & $\begin{array}{c}\text { Exercise Science }(5 \%) \\
\text { Business Administration } \\
(4 \%) \\
\text { Criminal Justice }(3 \%) \\
\text { Accounting }(2 \%) \\
\text { Computer Science }(2 \%) \\
\text { Physical Education }(1 \%)\end{array}$ & Computer Science $(2 \%)$ & Sport Management (2\%) \\
\hline
\end{tabular}


Table 7

Most Common Academic Majors Among Student-Athletes

\begin{tabular}{ccccccccc}
\hline & \multicolumn{2}{c}{$\mathbf{2 0 0 9 - 2 0 1 0}$} & \multicolumn{2}{c}{$\mathbf{2 0 1 0 - 2 0 1 1}$} & \multicolumn{2}{c}{ 2011-2012 } & \multicolumn{2}{c}{ 2012-2013 } \\
& MEAC & CIAA & MEAC & CIAA & MEAC & CIAA & MEAC & CIAA \\
Sport Management & 22 & 61 & 28 & 80 & 40 & 78 & 32 & 99 \\
Criminal Justice & 26 & 27 & 33 & 50 & 40 & 42 & 44 & 105 \\
Business Management & 28 & 20 & 32 & 27 & 40 & 20 & 40 & 31 \\
\hline
\end{tabular}

Table 8

Most Common Areas of Study Among Student-Athletes

\begin{tabular}{cc} 
Area of Study & Percentage of Student-Athletes \\
Exercise, Sports, Kinesiology & $17 \%$ \\
Business & $15 \%$ \\
Social Sciences & $15 \%$ \\
Engineering & $8 \%$ \\
Communications & $6 \%$ \\
Biological Sciences & $3 \%$ \\
Other Academic Field & $3 \%$ \\
Education & $2 \%$ \\
Humanities and Fine Arts & $2 \%$ \\
Professional Studies & $2 \%$ \\
Physical Sciences and Mathematics & $1 \%$ \\
\hline
\end{tabular}

Table 9

Most Common Areas of Study Among Student-Athletes by Conference

\begin{tabular}{ccc}
\hline & $\begin{array}{c}\text { MEAC } \\
\text { (Division 1) }\end{array}$ & $\begin{array}{c}\text { CIAA } \\
\text { (Division 2) }\end{array}$ \\
$\begin{array}{c}\text { Exercise, Sports } \\
\text { Kinesiology }\end{array}$ & $14 \%$ & $19 \%$ \\
Business & $13 \%$ & $17 \%$ \\
Social Sciences & $15 \%$ & $14 \%$ \\
\hline
\end{tabular}


Table 10

Mid-Eastern Athletic Conference Football Area of Study

\begin{tabular}{|c|c|c|c|c|}
\hline & 2009 & 2010 & 2011 & 2012 \\
\hline University 1 & N/A & N/A & $\begin{array}{c}\text { Exercise, Sports, \& } \\
\text { Kinesiology }(19 \%) \\
\text { Business }(13 \%) \\
\text { Social Sciences }(11 \%)\end{array}$ & $\begin{array}{c}\text { Exercise, Sports, \& } \\
\text { Kinesiology }(19 \%) \\
\text { Social Sciences (13\%) } \\
\text { Business }(11 \%)\end{array}$ \\
\hline University 2 & $\begin{array}{l}\text { Social Sciences } \\
\quad(17 \%) \\
\text { Exercise, Sports, \& } \\
\text { Kinesiology }(15 \%) \\
\text { Business }(7 \%)\end{array}$ & $\begin{array}{c}\text { Social Sciences }(32 \%)^{*} \\
\text { Exercise, Sports, \& } \\
\text { Kinesiology }(14 \%) \\
\text { Business }(14 \%)\end{array}$ & $\begin{array}{l}\text { Social Sciences }(23 \%) \\
\text { Business }(14 \%) \\
\text { Engineering, } \\
\text { Computer/Information } \\
\text { Sciences }(10 \%)\end{array}$ & $\begin{array}{c}\text { Social Sciences }(30 \%)^{*} \\
\text { Exercise, Sports, \& } \\
\text { Kinesiology }(18 \%) \\
\text { Business }(18 \%)\end{array}$ \\
\hline University 3 & $\begin{array}{l}\text { No Football } \\
\text { Program }\end{array}$ & No Football Program & No Football Program & No Football Program \\
\hline University 4 & N/A & $\begin{array}{c}\text { Unlisted/Undecided } \\
(74 \%)\end{array}$ & Unlisted/Undecided (70\%) & Unlisted/Undecided (79\%) \\
\hline University 5 & $\begin{array}{c}\text { Exercise, Sports, \& } \\
\text { Kinesiology }(19 \%) \\
\text { Social Sciences } \\
(17 \%) \\
\text { Business }(17 \%)\end{array}$ & $\begin{array}{c}\text { Exercise, Sports, \& } \\
\text { Kinesiology }(19 \%) \\
\text { Engineering, } \\
\text { Computer/Information } \\
\text { Sciences }(17 \%) \\
\text { Business }(17 \%)\end{array}$ & $\begin{array}{c}\text { Exercise, Sports, \& } \\
\text { Kinesiology }(20 \%) \\
\text { Business }(14 \%) \\
\text { Social Sciences }(12 \%)\end{array}$ & $\begin{array}{c}\text { Social Sciences }(23 \%) \\
\text { Exercise, Sports, \& } \\
\text { Kinesiology }(15 \%) \\
\text { Engineering, } \\
\text { Computer/Information } \\
\text { Sciences }(13 \%)\end{array}$ \\
\hline University 6 & N/A & $\begin{array}{c}\text { Unlisted/Undecided } \\
(84 \%)\end{array}$ & Unlisted/Undecided (58\%) & Unlisted/Undecided (53\%) \\
\hline University 7 & $\begin{array}{c}\text { Social Sciences } \\
(21 \%) \\
\text { Business }(20 \%) \\
\text { Exercise, Sports, \& } \\
\text { Kinesiology }(14 \%)\end{array}$ & $\begin{array}{l}\text { Social Sciences }(28 \%)^{*} \\
\text { Exercise, Sports, \& } \\
\text { Kinesiology }(24 \%) \\
\text { Business }(19 \%)\end{array}$ & $\begin{array}{l}\text { Social Sciences }(22 \%) \\
\text { Business }(20 \%) \\
\text { Exercise, Sports, \& } \\
\text { Kinesiology }(19 \%)\end{array}$ & $\begin{array}{l}\text { Social Sciences }(19 \%) \\
\text { Exercise, Sports, \& } \\
\text { Kinesiology }(19 \%) \\
\text { Business }(18 \%)\end{array}$ \\
\hline University 8 & $\begin{array}{l}\text { Unlisted/Undecided } \\
(83 \%)\end{array}$ & N/A & N/A & N/A \\
\hline University 9 & N/A & $\begin{array}{c}\text { Unlisted/Undecided } \\
\quad(37 \%) \\
\text { Exercise, Sports, \& } \\
\text { Kinesiology }(21 \%) \\
\text { Social Sciences }(15 \%)\end{array}$ & $\begin{array}{c}\text { Unlisted/Undecided (44\%) } \\
\text { Exercise, Sports, \& } \\
\text { Kinesiology }(21 \%) \\
\text { Social Sciences }(11 \%)\end{array}$ & $\begin{array}{c}\text { Unlisted/Undecided (29\%) } \\
\text { Social Sciences }(18 \%) \\
\text { Business }(13 \%)\end{array}$ \\
\hline
\end{tabular}


Table 11

Central Intercollegiate Athletic Association Football Area of Study

\begin{tabular}{|c|c|c|c|c|}
\hline & 2009 & 2010 & 2011 & 2012 \\
\hline University 10 & $\begin{array}{c}\text { Business }(29 \%)^{*} \\
\text { Social Sciences } \\
(24 \%) \\
\text { Communications } \\
(9 \%)\end{array}$ & $\begin{array}{c}\text { Business }(26 \%)^{*} \\
\text { Social Sciences }(18 \%) \\
\text { Exercise, Sports, \& } \\
\text { Kinesiology }(15 \%)\end{array}$ & $\begin{array}{c}\text { Business }(27 \%)^{*} \\
\text { Social Sciences }(20 \%) \\
\text { Exercise, Sports, \& } \\
\text { Kinesiology }(15 \%)\end{array}$ & $\begin{array}{c}\text { Business }(28 \%)^{*} \\
\text { Exercise, Sports, \& } \\
\text { Kinesiology }(18 \%) \\
\text { Social Sciences }(12 \%)\end{array}$ \\
\hline University 11 & $\begin{array}{c}\text { Exercise, Sport, \& } \\
\text { Kinesiology }(34 \%)^{*} \\
\text { Social Sciences } \\
(18 \%) \\
\text { Business }(17 \%)\end{array}$ & $\begin{array}{c}\text { Exercise, Sport, \& } \\
\text { Kinesiology }(38 \%)^{*} \\
\text { Business }(20 \%) \\
\text { Social Sciences }(17 \%)\end{array}$ & $\begin{array}{c}\text { Exercise, Sport, \& } \\
\text { Kinesiology (30\%)* } \\
\text { Social Sciences (18\%) } \\
\text { Business }(15 \%)\end{array}$ & $\begin{array}{c}\text { Exercise, Sport, \& } \\
\text { Kinesiology }(23 \%) \\
\text { Social Sciences }(23 \%) \\
\text { Business }(14 \%)\end{array}$ \\
\hline University 12 & N/A & $\begin{array}{c}\text { Unlisted/Undecided } \\
(38 \%) \\
\text { Social Sciences }(19 \%) \\
\text { Business }(16 \%)\end{array}$ & N/A & $\begin{array}{c}\text { Unlisted/Undecided }(28 \%) \\
\text { Social Sciences }(21 \%) \\
\text { Exercise, Sports, \& } \\
\text { Kinesiology }(19 \%)\end{array}$ \\
\hline University 13 & $\begin{array}{c}\text { Unlisted/Undecided } \\
\qquad(31 \%) \\
\text { Exercise, Sports, \& } \\
\text { Kinesiology }(24 \%) \\
\text { Business }(15 \%)\end{array}$ & $\begin{array}{c}\text { Exercise, Sports, \& } \\
\text { Kinesiology }(36 \%)^{*} \\
\text { Business }(16 \%) \\
\text { Engineering, } \\
\text { Computer/Information } \\
\text { Sciences }(16 \%)\end{array}$ & $\begin{array}{c}\text { Exercise, Sports, \& } \\
\text { Kinesiology }(29 \%)^{*} \\
\text { Engineering, } \\
\text { Computer/Information } \\
\text { Sciences }(13 \%)\end{array}$ & $\begin{array}{c}\text { Exercise, Sports, \& } \\
\text { Kinesiology }(31 \%)^{*} \\
\text { Engineering, } \\
\text { Computer/Information } \\
\text { Sciences }(13 \%) \\
\text { Business }(10 \%)\end{array}$ \\
\hline University 14 & N/A & $\begin{array}{c}\text { Unlisted/Undecided } \\
(52 \%) \\
\text { Business }(15 \%) \\
\text { Social Sciences }(8 \%)\end{array}$ & $\begin{array}{c}\text { Unlisted/Undecided } \\
(64 \%) \\
\text { Social Sciences }(10 \%) \\
\text { Professional Studies }(6 \%)\end{array}$ & $\begin{array}{c}\text { Social Sciences (21\%) } \\
\text { Business (14\%) } \\
\text { Professional Studies (11\%) }\end{array}$ \\
\hline University 15 & N/A & $\begin{array}{c}\text { Exercise, Sports, \& } \\
\text { Kinesiology }(27 \%)^{*} \\
\text { Social Sciences }(13 \%) \\
\text { Engineering, } \\
\text { Computer/Information } \\
\text { Sciences }(13 \%)\end{array}$ & $\begin{array}{c}\text { Other Academic Field } \\
(27 \%)^{*} \\
\text { Exercise, Sports, \& } \\
\text { Kinesiology }(24 \%) \\
\text { Business }(15 \%)\end{array}$ & $\begin{array}{c}\text { Exercise, Sports, \& } \\
\text { Kinesiology }(28 \%)^{*} \\
\text { Social Sciences }(15 \%) \\
\text { Engineering, } \\
\text { Computer/Information } \\
\text { Sciences }(13 \%)\end{array}$ \\
\hline University 16 & $\mathrm{~N} / \mathrm{A}$ & N/A & N/A & $\begin{array}{c}\text { Exercise, Sports, \& } \\
\text { Kinesiology }(28 \%)^{*} \\
\text { Social Sciences }(20 \%) \\
\text { Business }(12 \%)\end{array}$ \\
\hline University 17 & $\mathrm{~N} / \mathrm{A}$ & N/A & N/A & $\begin{array}{c}\text { Social Sciences }(43 \%)^{* *} \\
\text { Business }(25 \%)^{*} \\
\text { Education }(10 \%)\end{array}$ \\
\hline University 18 & $\begin{array}{c}\text { Unlisted/Undecided } \\
(29 \%) \\
\text { Business }(29 \%)^{*} \\
\text { Exercise, Sport, \& } \\
\text { Kinesiology }(19 \%)\end{array}$ & $\begin{array}{c}\text { Unlisted/Undecided } \\
(60 \%) \\
\text { Business }(16 \%) \\
\text { Exercise, Sport, \& } \\
\text { Kinesiology }(12 \%)\end{array}$ & $\begin{array}{c}\text { Unlisted/Undecided } \\
(51 \%) \\
\text { Business }(20 \%) \\
\text { Exercise, Sport, \& } \\
\text { Kinesiology (9\%) }\end{array}$ & $\begin{array}{c}\text { Unlisted/Undecided (81\%) } \\
\text { Business (8\%) } \\
\text { Engineering, } \\
\text { Computer/Information } \\
\text { Sciences }(5 \%)\end{array}$ \\
\hline
\end{tabular}


Table 12

Mid-Eastern Athletic Conference Basketball Area of Study

\begin{tabular}{|c|c|c|c|c|}
\hline & $2009-2010$ & 2010-2011 & 2011-2012 & 2012-2013 \\
\hline University 1 & $\begin{array}{c}\text { Unlisted/Undecided } \\
(50 \%) \\
\text { Business }(25 \%)^{*}\end{array}$ & $\begin{array}{c}\text { Unlisted/Undecided } \\
(50 \%) \\
\text { Business }(17 \%)\end{array}$ & $\begin{array}{c}\text { Exercise, Sports, \& } \\
\text { Kinesiology }(33 \%)^{*} \\
\text { Communications }(25 \%)^{*}\end{array}$ & $\begin{array}{c}\text { Exercise, Sports, \& } \\
\text { Kinesiology }(40 \%)^{* *} \\
\text { Communications }(20 \%)\end{array}$ \\
\hline University 2 & $\begin{array}{l}\text { Unlisted/Undecided } \\
\quad(43 \%) \\
\text { Exercise, Sports, \& } \\
\text { Kinesiology }(29 \%)^{*}\end{array}$ & $\begin{array}{c}\text { Exercise, Sports, \& } \\
\text { Kinesiology }(50 \%)^{* *} \\
\text { Business }(19 \%)\end{array}$ & $\begin{array}{c}\text { Exercise, Sports, \& } \\
\text { Kinesiology }(53 \%)^{* *} \\
\text { Social Sciences }(20 \%)\end{array}$ & $\begin{array}{c}\text { Exercise, Sports, \& } \\
\text { Kinesiology }(43 \%)^{* *} \\
\text { Social Sciences }(21 \%)\end{array}$ \\
\hline University 3 & $\begin{array}{l}\text { Business }(39 \%)^{*} \\
\text { Social Sciences } \\
\quad(23 \%)\end{array}$ & $\begin{array}{c}\text { Business }(29 \%)^{*} \\
\text { Social Sciences }(21 \%)\end{array}$ & $\begin{array}{c}\text { Unlisted/Undecided } \\
(39 \%) \\
\text { Business (15\%) } \\
\text { Exercise, Sports, \& } \\
\text { Kinesiology (15\%) } \\
\text { Other Academic Field } \\
(15 \%)\end{array}$ & $\begin{array}{c}\text { Social Sciences }(29 \%)^{*} \\
\text { Business }(21 \%) \\
\text { Other Academic Field } \\
(21 \%)\end{array}$ \\
\hline University 4 & $\begin{array}{c}\text { Unlisted/Undecided } \\
(67 \%) \\
\text { Business }(23 \%)\end{array}$ & $\begin{array}{c}\text { Unlisted/Undecided } \\
(60 \%)\end{array}$ & $\begin{array}{c}\text { Unlisted/Undecided } \\
(43 \%)\end{array}$ & Unlisted/Undecided (54\%) \\
\hline University 5 & $\begin{array}{l}\text { Communications } \\
\qquad(15 \%) \\
\text { Exercise, Sports, \& } \\
\text { Kinesiology }(15 \%) \\
\text { Other Academic } \\
\text { Field }(15 \%)\end{array}$ & $\begin{array}{c}\text { Social Sciences }(25 \%)^{*} \\
\text { Business }(25 \%)^{*}\end{array}$ & $\begin{array}{l}\text { Social Sciences }(26 \%)^{*} \\
\text { Business }(26 \%)^{*}\end{array}$ & $\begin{array}{c}\text { Business }(26 \%)^{*} \\
\text { Communications }(20 \%)\end{array}$ \\
\hline University 6 & $\begin{array}{l}\text { Social Sciences } \\
\quad(20 \%) \\
\text { Business }(20 \%)\end{array}$ & $\begin{array}{c}\text { Engineering, } \\
\text { Computer/Information } \\
\text { Sciences }(18 \%) \\
\text { Exercise, Sports, \& } \\
\text { Kinesiology }(18 \%) \\
\text { Social Sciences }(18 \%)\end{array}$ & $\begin{array}{c}\text { Unlisted/Undecided } \\
(21 \%) \\
\text { Business }(21 \%)\end{array}$ & $\begin{array}{l}\text { Business }(33 \%)^{*} \\
\text { Exercise, Sports, \& } \\
\text { Kinesiology }(20 \%)\end{array}$ \\
\hline University 7 & $\begin{array}{l}\text { Social Sciences } \\
\qquad(36 \%)^{*}\end{array}$ & $\begin{array}{c}\text { Unlisted/Undecided } \\
(26 \%) \\
\text { Social Sciences (32\%)* }\end{array}$ & $\begin{array}{c}\text { Social Sciences }(33 \%)^{*} \\
\text { Unlisted/Undecided } \\
(20 \%)\end{array}$ & $\begin{array}{l}\text { Social Sciences }(27 \%)^{*} \\
\text { Exercise, Sports, \& } \\
\text { Kinesiology }(20 \%) \\
\text { Business }(20 \%)\end{array}$ \\
\hline University 8 & $\begin{array}{c}\text { Engineering, } \\
\text { Computer/Informatio } \\
\text { n Sciences }(31 \%)^{*} \\
\text { Physical Sciences \& } \\
\text { Mathematics }(19 \%)\end{array}$ & $\begin{array}{c}\text { Unlisted/Undecided } \\
(25 \%) \\
\text { Business }(19 \%) \\
\text { Communications }(19 \%) \\
\text { Engineering, } \\
\text { Computer/Information } \\
\text { Sciences }(19 \%)\end{array}$ & $\begin{array}{c}\text { Business }(27 \%)^{*} \\
\text { Biological Sciences } \\
(13 \%) \\
\text { Communications }(13 \%) \\
\text { Engineering, } \\
\text { Computer/Information } \\
\text { Sciences }(13 \%)\end{array}$ & $\begin{array}{c}\text { Unlisted/Undecided (31\%) } \\
\text { Business (23\%) } \\
\text { Communications (23\%) }\end{array}$ \\
\hline University 9 & N/A & $\begin{array}{l}\text { Business Management } \\
(25 \%)^{*} \\
\text { Social Sciences }(25 \%)^{*}\end{array}$ & $\begin{array}{c}\text { Exercise, Sports, \& } \\
\text { Kinesiology }(38 \%)^{*} \\
\text { Social Sciences }(38 \%)^{*}\end{array}$ & N/A \\
\hline
\end{tabular}




\section{Table 13}

Central Intercollegiate Athletic Association Basketball Area of Study

\begin{tabular}{|c|c|c|c|c|}
\hline & 2009-2010 & $2010-2011$ & 2011-2012 & 2012-2013 \\
\hline $\begin{array}{l}\text { University } \\
10\end{array}$ & $\begin{array}{c}\text { Communications }(33 \%)^{*} \\
\text { Social Sciences }(17 \%) \\
\text { Business }(17 \%) \\
\text { Exercise, Sports, \& } \\
\text { Kinesiology }(17 \%)\end{array}$ & $\begin{array}{c}\text { Communications } \\
(29 \%)^{*} \\
\text { Business }(21 \%)\end{array}$ & $\begin{array}{c}\text { Communications }(50 \%)^{* *} \\
\text { Business }(29 \%)^{*}\end{array}$ & $\begin{array}{c}\text { Communications }(43 \%)^{* *} \\
\text { Business }(29 \%)^{*}\end{array}$ \\
\hline $\begin{array}{l}\text { University } \\
11\end{array}$ & $\begin{array}{c}\text { Exercise, Sports, \& } \\
\text { Kinesiology }(38 \%)^{*} \\
\text { Social Sciences }(25 \%)^{*}\end{array}$ & N/A & N/A & $\begin{array}{c}\text { Business (36\%)* } \\
\text { Exercise, Sports, \& } \\
\text { Kinesiology (29\%)* }\end{array}$ \\
\hline $\begin{array}{l}\text { University } \\
12\end{array}$ & N/A & $\begin{array}{l}\text { Business }(33 \%)^{*} \\
\text { Social Sciences } \\
\quad(20 \%)\end{array}$ & $\begin{array}{c}\text { Business }(27 \%)^{*} \\
\text { Social Sciences }(20 \%)\end{array}$ & $\begin{array}{c}\text { Business }(27 \%)^{*} \\
\text { Biological Sciences }(20 \%) \\
\text { Social Sciences }(20 \%)\end{array}$ \\
\hline $\begin{array}{c}\text { University } \\
13\end{array}$ & $\begin{array}{c}\text { Exercise, Sports, \& } \\
\text { Kinesiology }(29 \%)^{*} \\
\text { Engineering, } \\
\text { Computer/Information Systems } \\
(14 \%) \\
\text { Social Sciences }(14 \%)\end{array}$ & $\begin{array}{l}\text { Exercise, Sports, \& } \\
\text { Kinesiology } \\
(50 \%)^{* *} \\
\text { Unlisted/Undecided } \\
(33 \%)\end{array}$ & $\begin{array}{c}\text { Exercise, Sports, \& } \\
\text { Kinesiology }(67 \%)^{* *} \\
\text { Unlisted/Undecided (17\%) }\end{array}$ & $\begin{array}{l}\text { Exercise, Sports, \& } \\
\text { Kinesiology }(69 \%)^{* *}\end{array}$ \\
\hline $\begin{array}{c}\text { University } \\
14\end{array}$ & $\begin{array}{c}\text { Business }(33 \%)^{*} \\
\text { Engineering, } \\
\text { Computer/Information Sciences } \\
(20 \%)\end{array}$ & $\begin{array}{l}\text { Business }(40 \%)^{* *} \\
\text { Engineering, } \\
\text { Computer/Informati } \\
\text { on Sciences }(20 \%)\end{array}$ & $\begin{array}{c}\text { Business (31\%)* } \\
\text { Biological Sciences (13\%) }\end{array}$ & $\begin{array}{c}\text { Business }(29 \%)^{*} \\
\text { Social Sciences }(21 \%)\end{array}$ \\
\hline $\begin{array}{l}\text { University } \\
15\end{array}$ & $\begin{array}{c}\text { Exercise, Sports, \& } \\
\text { Kinesiology }(38 \%)^{*} \\
\text { Social Sciences }(25 \%)^{*}\end{array}$ & $\begin{array}{c}\text { Exercise, Sports, \& } \\
\text { Kinesiology } \\
(50 \%)^{* *} \\
\text { Social Sciences } \\
(13 \%)\end{array}$ & $\begin{array}{c}\text { Exercise, Sports, \& } \\
\text { Kinesiology (39\%)* } \\
\text { Other Academic Field } \\
\qquad(17 \%)\end{array}$ & $\begin{array}{c}\text { Exercise, Sports, \& } \\
\text { Kinesiology }(36 \%)^{*} \\
\text { Business }(14 \%)\end{array}$ \\
\hline $\begin{array}{c}\text { University } \\
16\end{array}$ & $\begin{array}{c}\text { Communications }(25 \%) * \\
\text { Business }(17 \%) \\
\text { Social Sciences }(17 \%)\end{array}$ & $\begin{array}{c}\text { Business }(31 \%)^{*} \\
\text { Exercise, Sports, \& } \\
\text { Kinesiology }(23 \%)\end{array}$ & $\begin{array}{l}\text { Exercise, Sports, \& } \\
\text { Kinesiology }(47 \%)^{* *} \\
\text { Business }(40 \%)^{* *}\end{array}$ & $\begin{array}{c}\text { Exercise, Sports, \& } \\
\text { Kinesiology }(33 \%)^{*} \\
\text { Business }(20 \%)\end{array}$ \\
\hline $\begin{array}{l}\text { University } \\
17\end{array}$ & Business $(40 \%)^{* *}$ & N/A & N/A & N/A \\
\hline $\begin{array}{c}\text { University } \\
18\end{array}$ & $\begin{array}{c}\text { Social Sciences }(25 \%)^{*} \\
\text { Business }(20 \%)\end{array}$ & $\begin{array}{c}\text { Unlisted/Undecided } \\
(38 \%) \\
\text { Social Sciences } \\
(19 \%)\end{array}$ & N/A & $\begin{array}{l}\text { Unlisted/Undecided (47\%) } \\
\text { Exercise, Sports, \& } \\
\text { Kinesiology }(12 \%) \\
\text { Social Sciences }(12 \%)\end{array}$ \\
\hline
\end{tabular}




\section{Appendix B}

\section{Introduction}

\section{Extended Review of Literature}

The structure of the education system in the United States is unlike any other country in the world (Chu, 1985). No other country has sport paired with education starting from the middle school levels and ending just short of a professional career at the intercollegiate level. Chu (1985) argued that United States higher education system is paired with sport because leaders of colleges and universities were not clear about their charter, an unspoken and informal understanding of the purposes and responsibilities of an institution, when they were created. Without clear objectives and purposes of higher education in the United States, there was a space for debate and discussion about new programs to include that differ from European higher education curriculum and models. It became clear that including sport in the formal structure of higher education would aid in attracting students and new funds for institutions (Chu, 1985). This notion has been proven true throughout history, as intercollegiate sport has been responsible for the integration of several different sectors of the American population into higher education from women and minorities to those low in socioeconomic status. At the beginning of the $20^{\text {th }}$ century, intercollegiate sport was firmly implanted into the structure of higher education and the hiring of full-time coaches, offering of scholarships, and formal schedules of competition amongst schools became the norm (Chu, 1985). Scholars who studied intercollegiate sport began to raise questions of the notion of exploitation of some student-athletes based on race and educational experience as intercollegiate sport became more visible nationwide (Edwards, 1985).

Clustering by academic major fits in the context of student-athlete exploitation in intercollegiate sport. Case et al. (1987) defined clustering by academic major as the dynamic that exists when $25 \%$ or more of the student-athletes on a team's roster pursue the same academic 
major. Clustering has been found to disproportionately affect African-American male studentathletes in basketball and football more than any other population of student-athletes. Despite the fact that graduation rates have increased for African-American male student-athletes in football and basketball, clustering by academic experience calls into question the quality of their academic experience. Furthermore, with the knowledge that student-athletes often have weaker entering skills (high school GPA, SAT/ACT scores) than non-athlete students, it has been posited that clustering by academic major is simply a phenomenon that allows student-athletes to remain eligible to participate in their sport and that without clustering by academic major, some student-athletes would not be able to gain admission to the college or university they represent in competition. Clustering has primarily been researched in NCAA Division I member institutions and conferences, largely because these institutions and conferences are among the most visible and most commercialized. The increased visibility and commercialization of some member institutions has led to more time demands and responsibilities on student-athletes to their sport. Some challenge the notion that student-athletes have equal educational opportunities as their non-athlete student peers. Dubois (1985) argued that participation in sport functions as a vehicle of social mobility is a myth. With Dubois's (1985), the argument that the student-athlete has similar education opportunities as non-athlete student peers and benefits from the athletic scholarship and other services made available to student-athletes loses strength.

Historically Black Colleges and Universities (HBCUs) are an area of study that has been neglected in much of the research about the student-athlete experience. Some HBCUs are NCAA member institutions across the three divisions. However, these institutions were often created with a special mission: to provide higher education to those who otherwise may not have access 
to higher education. Therefore, HBCUs have a history of admitting students whose entering skills and test scores are weaker than those attending other institutions.

Data suggests that clustering by academic major exists at larger institutions where student-athletes may not have the entering skills to succeed at the college, it is important to understand if clustering by academic major exist at HBCUs where students are admitted and known to not have the entering skills of their peers at predominantly white institutions (PWIs). One of the missions of these institutions is to nurture and teach black students in a way that is different than PWIs. If clustering by academic major among student-athletes exists at these institutions, there are major implications about the importance of academics for student-athletes at these institutions and any institution of higher education.

\section{The History of Historically Black Colleges and Universities (HBCUs)}

Historically Black Colleges and Universities (HBCUs) are often thought of as exclusively educating blacks, but they have served as a major part of the nation's higher education system since the end of slavery and the Civil War. Immediately following the Civil War, the Freedmen's Bureau, Black religious organizations, and church missionaries were left with the responsibility to educate black citizens just freed from enslavement (Exkano, 2013). Because blacks were denied the opportunity to obtain a formal education throughout slavery, many blacks valued the pursuit of formal education as a way of HBCUs were established to provide education for blacks who were prohibited from receiving formal higher education at other institutions across the country (Nichols, 2004). After the Emancipation Proclamation, two Morrill Acts were passed to create agricultural and mechanical colleges as society shifted towards that type of education (Exkano, 2013). The first Morrill Land-Grant Act was passed in 1862, but the second Morrill Land-Grant Act, passed in 1890, strictly enforced building colleges for only black students in the 
face of southern segregation (Exkano, 2013). Unfortunately, many of these colleges fell victim to less than adequate funding, which affected many parts of the institutions such as facilities and faculty salaries (Esters \& Strayhorn, 2013; Exkano, 2013) and this less than adequate funding still exists today (Brown II, 2013). Therefore, many HBCUs initial mission is unique when compared to other higher education institutions (Nichols, 2004). HBCUs are also known for opening their doors to poor whites, women, and other marginalized groups of people when they did not have access to formal higher education (Redd, 1998; Allen, Jewell, Griffin, and Wolf, 2007; Stevenson, 2007; Esters \& Strayhorn, 2013).

Enrollment at HBCUs has risen and fallen with the times and was affected by the Civil Rights Act, attrition of black students from PWIs, and the overall college going patterns of black students (Brown II, 2013; Nichols, 2004). The decrease in enrollment from black students has increased due to students of other racial and ethnic groups choosing to attend HBCUs as well as an increase in first generation college students (Esters \& Strayhorn, 2013; Nichols, 2004). Although over $200 \mathrm{HBCUs}$ were founded before 1890, today there are 103 and they make up about 3\% of all postsecondary institutions (Esters \& Strayhorn, 2013; Nichols, 2004).

According to Cantey, Bland, Mack, \& Joy-Davis (2013), Nichols (2004), and Allen et al. (1991), HBCUs have six goals: "maintaining the Black American historical and cultural tradition; providing key leadership for the Black American community; providing Black American role models for social, political, and economic purposes in the Black community; assuring economic function in the Black American community; providing Black American role models for social, political, and economic purposes in the Black community to address issues between minority and majority populations; producing Black agents for research, institutional training, and information dissemination in the Black and other minority communities." 
HBCUs are woefully understudied as separate entities; instead they are frequently compared to predominantly white institutions (PWI) (Brown II, 2013). Researchers have compared the experiences and outcomes of black students at PWIs, studied the experiences and outcomes of white students at HBCUs, and compared the resources that HBCUs have to many PWIs (Esters \& Strayhorn, 2013; Palmer, Davis, \& Maramba, 2010). Also, in the face of an increasingly diverse ethnic minority society, their relevance and pertinence are often questioned and have been called into question by critics and scholars (Brown II, 2013; Cantey et al., 2013; Esters \& Strayhorn, 2013; Exkano, 2013). Exkano (2013) asserted that the narratives about HBCUs stem from a Western perspective, which tends to distort stories and experiences in a manner that casts these narratives as negative events. This comparison serves as a disservice to these institutions as they have just as much variety as PWIs in regards to size, specialized curriculum, traditions, etc. (Brown II, 2013).

\section{NCAA Division II}

Jay Coakley (2004), known scholar and sport sociologist, concluded that athletic talent is usually higher in National Collegiate Athletic Association (NCAA) member institutions that compete at the Division I level than Division II or Division III levels (Coakley, 2004). Coakley (2004) elaborates on the differences between Division I athletics and other divisions, citing the amount of traveling, media coverage, and consequences of winning and losing. In a more recent update to his text, Coakley (2009) did not address the differing talent level in NCAA divisions, but only acknowledged that NCAA Divisions II and III are less big-time than NCAA Division I. Coakley's assertion about the talent level among NCAA Divisions is a common assumption, but the talent level is not the sole reason the NCAA member institutions are divided into three divisions. 
The true difference in NCAA divisions is based on the number of sports and the amount of funding available to student-athletes. While Division I programs often boast large numbers of full athletic scholarships, Division II and Division III programs do not have as many athletic scholarships. Division II programs can offer athletic scholarships to future students, but any financial aid that a student-athlete receives in a Division III athletic program must come from an academic financial aid package. The Division II athletic experience is advertised as an intermediate level of competition between Division I and Division III because of the ability to receive athletic scholarships, just not at the probability of Division I athletics. The Division III athletic experience is intended to focus more on the social well being of the student-athlete than maintaining the billion-dollar industry of Division I athletics. Even an NCAA advertisement advocates that the Division III athletic experience is intended to focus on student-athletes who "play for the love of the game, without the obligation of an athletic scholarship." (NCAA, 2013a) Existing misconceptions and misunderstandings about Division II and Division III athletics are exacerbated by a limited number of research articles published about the Division II and Division III student-athlete experience. These research articles are limited to athletic identity, defribilator use, and burnout (Judge et al., 2012; Drezners, Rogers, \& Horneff, 2010; Sturan, Feltz, \& Gilson, 2011). NCAA Division I is the most prominent and well-known division. In revenue generating sports, NCAA Division I competition receive much more media coverage and attention than either of the other two NCAA divisions.

One issue with NCAA Division II data is that it is often compared with NCAA Division I data. The prominence and visibility of NCAA Division I athletics make it the standard structure for intercollegiate athletics; however, the comparison interferes with the ability to understand the Division II environment as its own. NCAA Division II data is compared to the other two NCAA 
Divisions data to frame the notion that the NCAA's Division II is an intermediate division, one with fewer resources and less visibility than NCAA Division I yet more resources and visibility than NCAA Division III. In order to compete in the NCAA's Division I, an institution must offer at least fourteen sports and at least two team sports for each gender. In order to offer a sport, these institutions must have adequate funding to cover expenses for travel and equipment, and coaches and satisfactory practice and competition facilities. Often, NCAA Division II institutions have significantly smaller budgets than NCAA Division I institutions due to the lack of television contracts and corporate sponsorships (Cooper \& Hawkins, 2012). These sports can be divided in one of two ways: seven men's sports and seven women's sports or six men's sports and eight women's sports. The NCAA's Division II requires an institution to offer at least ten sports. These sports can be divided into five men's sports and five women's sports or four men's sports and six women's sports. The NCAA's Division III requires an institution to offer at least five sports for men and five sports for women (NCAA, 2011a). There are 302 Division II member institutions, 33 fewer than the number of Division I member institutions. The average enrollment at a Division II member institution is 4,500 students, and the sizes of these institutions range from less than 2,500 students to over 15,000 students (NCAA, 2011b).

On the NCAA's Division II website, a personal data file (PDF) entitled "Division II Elevator Speech" emphasizes the balance that student-athletes can receive by choosing to compete at the Division II level instead of the Division I level. The file emphasizes the following tenets of Division II athletics: academic success, the skill level of Division II recruits, community engagement, and fiscally responsible athletic departments. Regarding academic success, the document cites that 73 percent of all Division II student-athletes, scholarship and non-scholarship, graduate within six years of their initial enrollment if they remain full-time 
students and that Division II student-athletes perform better academically than non-athlete students. In fact, the gap between the Division II student-athlete graduation rate and that of nonathlete students is larger than the gap that exists between the student-athletes and non-athlete students at NCAA Division I and Division III levels. Regarding the skill level of Division II student-athletes, the document cites increased national television exposure for Division II championships since 2000-2001 and the fact that nearly 50 percent of Division II student-athletes receive athletic financial aid for their participation. Finally, the document cites that Division II institutions have worked to be more welcoming to communities surrounding those campuses and that it costs an institution less than 50 percent of the money it would cost to run a Division I athletics program (NCAA, 2011c).

To date, studies that analyze student-athlete experience at Division II institutions are limited. Baucom and Lantz (2001) studied faculty members at a highly academically prestigious Division II institution to compare their attitudes toward student-athletes to their non-athlete student peers. The researchers found that the faculty members held prejudicial attitudes toward student-athletes more than toward their non-athlete student peers in the following regards: special admissions policies, student-athletes receiving a full scholarship without the scholarship being denoted as academic or athletic, special academic support and services for student-athletes, and coverage of student-athlete competitions in the campus newspaper. The researchers also found that the prejudicial attitudes were not limited to student-athletes competing in revenuegenerating sports. However, the researchers failed to recognize whether the negative faculty attitudes ever manifested themselves in specific behaviors towards the student-athletes. Although the findings cannot be generalized to all Division II institutions, the findings and other research suggest that prejudicial attitudes that may exist from faculty members toward student-athletes at 
more prominent Division I institutions also exist at Division II institutions (Engstrom \& Sedlacek, 1995).

Nite (2012) studied one Division II athletic department's perspective on the challenges, such as identity development and time management that accompany student-athlete development. The athletic director in the study revealed that part of the challenge in holistically supporting his student-athletes was rooted in limited resources. Larger institutions, namely division I, may have the resources that Division II institutions lack to hire more personnel to assist different aspects of student-athlete development_-academic, social, and in some cases, spiritual. Interestingly enough, resources, or lack thereof, are at the center of the debate around HBCUs, their history, and their current purpose and relevance.

\section{Historically Black Colleges and Universities in Athletics}

In addition to their academic, social, and cultural support, HBCUs were the only places that black students could compete in collegiate athletics until the middle of the $20^{\text {th }}$ century (Hodge, Bennett III, and Collins, 2013). The Georgia-Carolina Athletic Association in 1910 and the Colored Intercollegiate Athletic Association (CIAA) in 1912 were the first organizational structures for HBCU athletics (Hodge et al., 2013). The National Association of Intercollegiate Athletics (NAIA) became the first predominantly white athletic association to admit HBCUs as members in 1953, and it took the NCAA over 10 years after Brown v. Board of Education to accept HBCUs as member institutions (Hodge et al., 2013). The admission of black or studentathletes into sports at predominantly white educational institutions put HBCUs at a disadvantage to recruit many of the top black student-athletes to their programs because many HBCUs simply did not have as much funding as some of the predominantly white educational institutions. Today, HBCUs and their athletic departments have not only been affected by the admission of 
black student-athletes to predominantly white educational institutions, but the passage of legislation such as Title IV, Proposition 48, and the NCAA Academic Progress Rate (APR) (Stuart, 2012; Hodges et al., 2013). Financial issues that affect HBCU athletic departments and the struggles to recruit top black student-athletes are issues that extend beyond athletics. Accreditation, shifting in college attendance patterns of black students, and discourse challenging the benefits and relevance of HBCUs are issues that these institutions face today (Allen, et al., 2007; Brown II, 2013; Charlton, 2011; Fester, Gasman, Nguyen, 2012).

HBCUs provide an environment that exists an intersection between many of the most common populations examined in sports studies - males, African-Americans, and revenuegenerating sport student-athletes. Although most HBCUs are members of NCAA Division II, III, or other collegiate sports organizations, there are two NCAA Division I conferences comprised of HBCUs - the Mid-Eastern Athletic Conference (MEAC) and the Southern Intercollegiate Athletic Conference (SIAC) (Hodge et al., 2013; Cooper \& Hawkins, 2012). Therefore, HBCUs provide a space to study general black student and black student-athlete experiences across all three NCAA divisions.

Cooper and Hawkins (2012) used quantitative and qualitative methods to learn more about the experiences of black male football and basketball players at an HBCU in the Southeastern United States. Cooper and Hawkins (2012) used the Student Athlete Questionnaire (SAQ) to obtain quantitative data for analysis and conducted a single focus group rooted in grounded theory for their qualitative analysis. The scholars posed three research questions to the participants $(n=48)$ that addressed the motivations for attending an HBCU, the holistic (academic, athletic, and social) experiences of these student-athletes, and the factors associated with studentathlete academic achievement at HBCUs (Cooper \& Hawkins, 2012). In order to be selected, the 
participants had to fit the following criteria: self-identification as black or African-American, active participants of the varsity football and/or men's basketball team, and current full-time enrollment status. The researchers found that student-athletes appreciated the opportunity to play and excel at an HBCU, viewed the opportunity to attend a HBCU as one to develop holistically, sought a community feel from the institution in the form of small classes, relationships with professors, and academic support programs, and believed that their social experiences were positively affected by their status as a student-athlete.

Charlton (2011) attempted to critique the athletic department organizational subculture on the campus of an HBCU. The primary purpose of this analysis was to better understand the role of the athletic department above average graduation rate for student-athletes. Significant differences were found in the policies, rituals, and language of the investigated athletic department as compared to less successful HBCU athletic departments. Policies such as required visits to the academic services center on campus for student-athletes, enforced study hall hours complete with student-athlete reports, and weekly freshman and transfer student meetings stood out to the researcher in his data analysis. Specifically, the athletic department also enforced their own attendance policy for student-athlete classes and worked very closely with the academic services department at the institution. This close working relationship is instrumental in the program's success as the student-athletes, academic support staff, and coaches and athletic administrators are able to share and retain information necessary to help the student-athlete achieve academic success. Charlton (2011) also found that there was common language in the athletic department among the student-athletes, coaches, academic support staff, and athletic administrative staff that instructed student-athletes how to conduct themselves outside of athletics and conveyed the caring attitude of those in the athletic department. Finally, rituals such 
as an end-of-year athletic banquet that recognizes student-athlete academic achievements and special recognition during halftime of basketball games were also found to play a role in the athletic department's success in positively orienting student-athletes for academic success. Student-athletes at the institution look forward to participation in these two events, which gives them an opportunity to be celebrated in a way separate from their sport participation. Ultimately, Charlton (2011) found that intentional policy, language, and rituals could work to positively affect student-athlete academic success when successfully implemented. While there were some characteristics unique to the university being studied, the same organizational structure could be adopted to other HBCU athletic departments that have struggled to provide adequate academic support to their student-athletes. It is important that more research is conducted to discover whether the case of this athletic department is common or an anomaly.

\section{Limitations and Future Directions of NCAA Division II Research}

While research about HBCUs and NCAA Division II exists in academia, the research is not as prominent as the research is about predominantly white institutions (PWIs) and NCAA Division I. HBCUs and NCAA Division II environments are often studied as special populations and compared to the results and findings of studies of PWIs and NCAA Division I institutions instead of purposely researching in these environments to solely gain a better understanding of their existence. A review of the literature revealed that there is currently a dearth of research on student-athlete experience in NCAA Divisions II and III. Current research regarding Division II student-athletes non-sport experience revealed that these student-athletes face similar issues as their division I peers - stigmas and prejudices from faculty as well as large administrative issues that make it difficult to provide valuable support (Simons, 2007). Regarding student-athlete experiences at HBCUs, the research reveals that it is possible for athletic departments to 
effectively facilitate academic success among their student athletes and that some black male student-athletes seek many different benefits from their athletic experience at the institution.

There are several future directions that research can take to uncover more information about NCAA Division II student-athlete experience and HBCU student-athlete experience. First, there simply needs to be more research conducted. The case study (Charlton, 2011) of the athletic department and focus groups of football and male basketball players are experimental designs that can be replicated and conducted at different institutions. Conducting a similar experiment at a different institution can be a great contribution to the knowledge of NCAA Division II student-athlete experience and HBCU student-athlete experience. Future research can also seek to gather more empirical data from student-athletes from either of these populations. Currently, a large amount of the research about HBCU and Division II student-athlete experience is anecdotal, leaving a gap to be filled in the research by data (Brown II, 2013). The instrumentation used in future research is less important than the notion that research needs to be conducted. As previously mentioned, research about HBCUs and NCAA Division II studentathlete experience can only enhance existing knowledge about student-athlete experience. It is important to continue to learn more about these experiences to help those in these overlooked populations.

\section{NCAA Academic Reform (Proposition 16, Proposition 48, Academic Progress Rate)}

Issues of academic nature have been prominent in intercollegiate athletics for decades, but the level of analysis has evolved through the years (Petr \& McArdle, 2012). Today, due to the type of data collected, the growing amount of research performed, and the money and fame surrounding intercollegiate and professional sport participation (Johnson, Wessel, \& Pierce, 2012; Morgan, 2012), these academic issues have seemingly become more visible. 
Petr and McArdle (2012) outlined the history of NCAA Academic Policy by dividing the history into four eras: 1906-1980, the 1980s, the 1990s, and the early 200s. The scholars posit that although efforts to collect student-athlete data have grown and become more systematic through time, NCAA academic research began with and has been focused on student-athlete initial eligibility (Petr \& McArdle, 2012). The first era of NCAA academic research did not consist of much research aside from the minimum GPA (grade point average) rule established in the 1960s (Petr \& McArdle, 2012). The NCAA did no research in a national or systematic way during this era (Petr \& McArdle, 2012). All of the research surrounding the minimum GPA rule was done at the institutional or conference level (Petr \& McArdle, 2012). The second era of NCAA academic research occurred in the 1980s and was highlighted by Proposition 48 . Proposition 48 required incoming student-athletes to have a 2.0 GPA, 700 SAT score, and 11 “core high school courses" (p. 150) to be eligible for athletic participation (Johnson et al., 2012). This proposition allowed test scores to be a deciding factor for NCAA eligibility, a purpose that the College Board argued was not the intent or design of the SAT (Hanford, 1985). The NCAA passed Proposition 48 with no empirical research to support the legislation, but may have been motivated by two larger events occurring in the nation during that time. First, several highprofile scandals in college athletics were revealed, particularly the revelation of a number of high-performing student-athletes who had completed several years in college but were found to be functionally illiterate (Petr \& McArdle, 2012). Second, there was a national movement to improve higher education across the board (Petr \& McArdle, 2012). Proposition 48 outlined a sliding scale of high school GPA and standardized test score requirements, and was co-sponsored by the American Council on Education (Petr \& McArdle, 2012). However, the proposition faced resistance by those who questioned how the legislation would affect low-income and minority 
students, particularly those who utilize intercollegiate athletics as a vehicle to a college education (Cunningham, 2012). Legal scholars have even analyzed the racial bias that these propositions can have against low-income and minority students (Davis, 1994, 1996; Emerick, 1997) Without data to address the issue, the NCAA formed a special committee, the NCAA Academic Performance Study (APS), to conduct research with its member institutions (Petr \& McArdle, 2012).

The NCAA APS had collected sufficient academic data for analysis around the year 1990, which began the third era of NCAA Academic Policy (Petr \& McArdle, 2012). In this era, the NCAA APS studied high school academic performance in regards to initial eligibility, college academic performance of student-athletes and continuing eligibility, and the best ways to measure team-level academic success (Petr \& McArdle, 2012). This era also saw the birth of the Initial Eligibility Clearinghouse (IEC), now called the NCAA Eligibility Center (NEC), and Proposition 16. The IEC allowed the NCAA to oversee and verify the eligibility of all incomingfreshmen student-athletes in 1994 (Petr \& McArdle, 2012). Proposition 16 replaced Proposition 48, but included two changes (Johnson et al., 2012). The first change was a sliding scale relationship between standardized test scores and high school GPA (a higher GPA allows a lower standardized test score and vice-versa) and the second change was in the number of core high school courses, an increase to 13 from 11 (Johnson et al., 2012). After the creation of the IEC, the NCAA implemented the Academic Performance Program (APP) in 2003, which mandated Division I institutions to submit academic data for each of their scholarship athletes (Petr \& McArdle, 2012). After the large amount of data collected and made available to analyze to the NCAA during this era, two key findings were revealed (Petr \& McArdle, 2012). First, high school grades are better predictors of collegiate academic success than standardized test scores, 
but a combination of high school grades and standardized test scores is a better predictor than either variable used in isolation (Petr \& McArdle, 2012). Second, demographic variables such as race/ethnicity and income are important variables to consider when analyzing academic data because different demographic groups have different distributions of scores; therefore, any standardized rule on test scores and grades will impact demographic groups differently and perhaps unequally (Petr \& McArdle, 2012). Petr \& McArdle (2012) also found that when setting academic policy, a test cut-score (as opposed to a range) (used in Proposition 16 and Proposition 48) led to test scores being weighed heavier in consideration than high school GPA. A heavier consideration of test scores leads to a lower likelihood of accurate prediction of student-athlete success and increases the likelihood of excluding certain demographic populations from eligibility. In fact, the NCAA Committee on Academic Performance (CAP) found that HBCUs have not been able to keep up with their initial APRs, and in some instances, regressed in their APRs due to factors such as resource support services, admissions profiles, mission, contest scheduling, high rates of administrative turnover, and early exemption from APR penalties (Paskus, 2012).

The time period of the early twenty-first century marks the fourth era of NCAA Academic Policy. The fourth era of NCAA Academic policy is undoubtedly highlighted by the creation of the Academic Progress Rate (APR) in April 2004 (Johnson et al., 2012). The APR differs from Propositions 48 and 16 and other legislation addressing graduation rates because it provides coaches, administrators, and the general public with data about team academic progress, instead of individual student-athlete academic progress, in real time, semester by semester (Johnson et al., 2012). The APR is only calculated for student-athletes receiving any amount of financial aid due to his or her athletic participation at the institution and the calculation is based 
upon eligibility and retention criteria (Johnson et al., 2012). Student-athletes are eligible to earn two APR points each semester and four APR points per year. One point is awarded for being academically eligible and another point is awarded when the student-athlete returns to school the semester following the calculation period (Johnson et al., 2012). Academic eligibility is dependent upon the minimum standards for academic progress towards degree based on GPA and credit hours completed (Johnson et al., 2012). The overall APR is calculated when the total number of points earned by each student-athlete on the team is divided by the total number of points possible for the team to earn and the quotient is multiplied by 1000 (Johnson et al, 2012). Teams are penalized when the APR falls below 925 (Johnson et al., 2012). However, the APR still disproportionately affects some institutions, such as HBCUs (Blackman, 2008)

The Medill Reports (Hollencamp, 2009) addressed the issue of clustering as a potential byproduct of NCAA legislation of the Academic Progress Rate (APR) and titled the article, "NCAA Academic Ratings may force students to choose between dreams." Despite the fact that clustering may not be inherently bad, the coverage surrounding it undoubtedly depicts the dynamic in a very negative light. Dr. Greg Primus, a former Division I football player, sharply criticized the APR because he believes that it will influence players to stay away from challenging classes and promote clustering (Hollencamp, 2009). This process could be exacerbated by the fact that the penalties for failing to meet APR standards grow harsher for institutions who do not meet the standard for consecutive years (Hollencamp, 2009). If a team fails to meet their APR standard for four consecutive years, the entire institution will be restricted from NCAA Division I membership (Hollencamp, 2009).

Scholars have highlighted that high school performance (GPA and standardized test scores) can predict freshman year academic performance for student-athletes, but it is not a 
strong predictor of graduation from college (Petr \& McArdle, 2012). Therefore, the NCAA should examine the academic experience of student-athletes while they are on campus more closely (Cunningham, 2012; Petr \& McArdle, 2012). Scholars have also highlighted the differences in academic performance based on subgroups such as race/ethnicity, type of sport (revenue vs. non-revenue), gender, first generation college student, and others (Petr \& McArdle, 2012). However, Fields (2012) asserted that the NCAA should reexamine their research questions for data collection to ensure that the data collected benefits the group most affected by this legislation, student-athletes. Fields (2012) argued that it is clear that for one reason or another, previous NCAA academic legislation has been successful in increasing student-athlete graduation rates and settling debates surrounding initial eligibility; however, it is important to ask whether graduation rates and progress towards a degree are the most important parts of research to benefit student-athletes. Instead, it is important to collect data about the studentathlete academic experience outside of their sport and even outside of the classroom as much learning that occurs in college can occur in those spaces (Fields, 2012).

It is clear that much of the focus on NCAA Academic Legislation addresses graduation rates. In fact, this sentiment was even supported by former NCAA president Myles Brand (Roach, 2004). It is important to note, graduation rates do not shed light on the academic experience of student-athletes surrounding student-faculty interactions, classroom experiences, interactions with peers, or career and identity development. Career and identity development is crucial to any student during their time in college, especially when choosing an academic major.

\section{Choosing a Major}

Although it happens at different points in a student's college career, every college student must make the major decision to declare a major area of study to pursue in order to receive their 
Bachelor's degree (Motmarquette, Cannings, \& Mahseredjian, 2002). The decision about one's major in college can be the first step towards their future career. Student satisfaction with his or her academic major can be determined by several factors throughout the college experience such as strong faculty support, social support and encouragement from friends and peers, satisfaction with available student advising and counseling in the major, and the possibility of the coursework being interesting and useful for their future career (Leach and Patall, 2013). Other factors that influence a student's decision to pursue one major are gender role identification, interests and values, perceived abilities (Galotti, 1999), decision-making styles, resources on college campuses such as websites, advisors, and peers, and parents or other relatives (Galotti, Ciner, Altenbaumer, Geerts, Rupp, Woulfe, 2006), desire for social mobility (Wolniak, Seifert, Reed, Pascarella, 2008), connection to job opportunities and career aspirations (Motmarquette et al., 2002), and objective and perceived fit and adaptability (Wessel, Ryan, Oswald, 2008).

Wessel et al. (2008) studied the differences between objective fit and perceived fit of students who have declared their academic major. The researchers also studied adaptability due to the increasing societal trend in adaptability being a key trait for success in today's work environments. An individual's adaptability can have an effect on the level of objective or perceived fit he or she needs to have positive outcomes. Objective fit is evaluated by objective measures of personality and environment while perceived fit is evaluated by the individual's thoughts and perceptions of how they mesh in the environment. Objective fit and perceived fit do not necessarily occur independent of each other. Wessel et al. (2008) conducted the study with one hundred and ninety-eight $(n=198)$ undergraduate students, who volunteered to participate in the study in order to receive course credit. The survey measured demographic information, commitment to their major, perceived major fit, objective fit based upon the Strong Interest 
Inventory, adaptability based upon three adaptability scales, institutional satisfaction, academic self-efficacy, and withdrawal behaviors based upon avoidable class absences. Overall, the results indicated that perceived fit and objective fit are not very strongly related to each other in majorrelated outcomes such as affective major commitment, GPA, institutional satisfaction, probability of major change, and avoidable absences. However, there were significant correlations between different outcomes. The results indicated that academic self-efficacy was significantly correlated with perceived fit, but not objective fit. Also, the results indicated that there is not a significant relationship between academic self-efficacy and objective major fit. The researchers suggested that this lack of relationship may indicate that a student needs to perceive that he or she fits in their major in order to believe in their ability to achieve academic success. As far as practical implications of the study, the researchers noted that adaptability is very important in understanding student affective outcomes. Students who reported having high adaptability reported feeling more satisfied with their institution, having higher GPAs, and are not as likely to change their major as those reporting low adaptability. However, the most significant suggestions from the implications of the study were those for academic counselors. Wessel et al. (2008) suggested that academic counselors should identify students with low adaptability and dedicate extra time and effort with them to find a major that fits their interests because of the possibility of them having less positive major-related outcomes such as higher GPA, commitment to major, and institutional satisfaction, than their peers who are more adaptable.

Leach and Patall (2013) studied the relationships among college student decision-making orientation and post-decision analysis surrounding student satisfaction with their academic major. The scholars outlined the differences between maximizing and satisficing as two orientations of 
decision-making. When maximizing, one bases the decision upon the best available option with the belief that their wants and needs can be perfectly aligned in their decision. This orientation makes it more likely that one will spend more time exploring their options in pursuit of the perfect one. In satisficing, one bases the decision upon the option that simply meets the requirements or is "good enough" (Leach and Patall, 2013, p. 416). Those who make decisions following this orientation are less likely to experience regret about their decisions even if new or better options present themselves in the future (Leach and Patall, 2013). The scholars noted that maximizers are likely to be less satisfied with their decisions despite the fact that the decision closely aligns with their wants and needs. In their study, Leach and Patall (2013) surveyed 378 juniors and seniors from a large, tier-one research university in the southwestern United States. Juniors and seniors were selected for the study because students at the university are required to declare a major by the start of their junior year. The survey was composed of 53 items and included demographic questions, a designed scale to measure counterfactual thinking, the Maximization scale, and the Academic Major Satisfaction scale (AMSS). It was hypothesized that students with who employed maximizing in their decision-making orientation would be more likely to experience counterfactual thinking, thinking where an individual considers alternatives after a decision has been made. The researchers also hypothesized that students who experienced greater counterfactual thinking would report less satisfaction with their decision in academic major. The results aligned with the researchers' hypotheses.

While each of the aforementioned factors generally influence a student's decision to pursue one major instead of another, special populations certainly face other factors that influence a student's decision to pursue a major. Student-athletes are considered a special population on college campuses because of their unique roles and responsibilities that come from 
their athletic participation (Gaston-Gayles, 2003). Often, student-athletes benefit from guidance to balance their demands as a student while fulfilling their athletic obligations (Gaston-Gayles, 2003) and this guidance can come from many sources, teammates, coaches, peers, athletic academic advisors, and family members, just to name a few. The influence of coaches and athletic academic advisors can be tremendously strong on a student-athlete's overall academic experience, particularly on the decision of which major to pursue (Brooks, Etzel, Ostrow, 1987; Gruber, 2003).

Athletic academic advisors are faced with several challenges that academic advisors of non-athlete students do not, and it is important for them to be cognizant of these challenges to most effectively do their job (Gruber, 2003). These challenges include navigating the on-campus academic and athletic climate, determining faculty and non-athlete student attitudes towards student-athletes, addressing personal developmental issues for student-athletes, and understanding sport-specific needs and concerns (Gruber, 2003). Also, the position of athletic academic advisor was initially created to monitor and ensure the eligibility of student-athletes and was usually held by former coaches, players, or other individuals who may not have had training or interest in considering all of the aforementioned challenges to advising studentathletes (Gaston-Gayles, 2003). One of the by-products of today's increased commercialization and high stakes of college athletics is steering student-athletes into courses and majors that would make it easier to fulfill athletic roles and responsibilities (Sharp \& Sheilley, 2008). This process has implications for the significance of the academic experience of the student-athlete, the question of exploitation of the student-athlete, and refers to a dynamic known as clustering by academic major. 


\section{Clustering By Academic Major}

Case et al. (1987) published the first study that exclusively referred to academic clustering. Before this groundbreaking study, other studies (Purdy et al., 1982; Adler \& Adler, 1985) referred to clustering; however, one of the limitations of these studies was the failure to systematically or empirically examine its existence. Purdy et al. (1982) analyzed data from student-athletes of Colorado State University over a ten-year period after stating that academic standards had been altered to better facilitate intercollegiate athletics. Purdy et al. (1982) gained access to these records through the university's admissions office and compared the data in two ways. First, the student-athletes were compared to the general student population. Second, the student-athletes were compared to each other using different sub-categories such as the type of sport. The sample was comprised of the data of 2,091 male and female student-athletes $(\mathrm{n}=2,091)$. The data contained information about the athlete's admissions data such as high school grade point average and test scores, college transcript, financial aid, and the number of years the athlete took to complete college. The researchers found that female student-athletes were more prepared for and achieved higher grades in college than their male counterparts. Student-athletes in male revenue-generating sports had the lowest scores in high school grade point average, college grade point average, and graduation rate.

Case et al. (1987) decided to examine clustering after one of the authors interviewed for a teaching position at a university and was told that he would teach in a major designed solely for student-athletes. According to the university president, the major was created because the poor graduation rates for student-athletes seemed to indicate that they were being exploited (Case et al., 1987). After this experience, the author that interviewed for the teaching position examined the majors of football and men's basketball student-athletes at a well-known university in the 
Southern United states and found that $80 \%$ of the basketball players were pursuing the same major and $40 \%$ of the football players were pursuing the same major while less than one percent of the non-athlete student body pursued that major.

Case et al. (1987) requested media guides for men's and women's basketball teams from the 1985-1986 season and collected media guides from $103(n=103)$ randomly selected colleges from NCAA Division I basketball. Media guides usually list the roster of a team and include each player's name, height, weight, year in college, and academic major. Case et al. (1987) operationally defined clustering for their study as occurring when 25 percent or more of the players on a team are pursuing the same major or if a higher proportion of student-athletes are pursuing a major than the rest of the student population. In addition to the media guides, the scholars sent a questionnaire to the department chair of the clustered majors and inquired about the total number of students in the major and total number of students in the entire university. The scholars received 77 men's and 53 women's media guides. The media guides revealed that $55(71 \%)$ of the men's teams and $27(51 \%)$ of the women's teams demonstrate clustering by academic major. Also, 28 of the 55 men's teams and 20 of the 27 women's teams that demonstrated clustering returned the follow-up questionnaire. Case et al. (1987) then compared the percentage of student-athlete students pursuing a major where clustering was present to the percentage of non-athlete students pursuing the same major.

Case et al. (1987) found that clustering was more common for black student-athletes, in high achieving schools (programs that finished in the top 20 rankings in the last three years), and at schools with strong academic reputations (Case et al., 1987). One possible explanation for the increased likelihood in clustering for student-athletes in these types of programs is the gap between their academic abilities and the demands of their athletic participation. For example, at 
schools with high achieving athletic programs, some student-athletes may be asked to dedicate more time to their sport to maintain that level of athletic success. If more time is dedicated to maintaining athletic success, there is less time to dedicate to academic success. In another example, schools with strong academic reputations may present academic rigor that could lead a student-athlete to compare the importance of his or her athletic eligibility and experience to their academic experience. If maintaining athletic eligibility is more important than a quality academic experience, then a student-athlete may be influenced to choose a major that provides less academic rigor.

\section{Reports of Clustering and Scandal}

Since Case et al.'s 1987 study of clustering, mostly non-scholarly sources have reported instances of clustering and its effects on different parts of NCAA member institutions - studentathletes, faculty members, and university administrators. Unfortunately, these reports have not only detailed the existence of clustering, but its consequences as well, such as student-athletes still being declared academically ineligible, coaches or athletic academic advisors heavily influencing student-athlete majors, and professors or entire academic departments being scrutinized (Suggs, 2003; Ganczaruk, 2004; Thamel, 2006; Steeg, 2008; Elfman, 2009; Associated Press, 2011; Ellis \& Wilson, 2014). The Chronicle of Higher Education (Suggs, 2003), The New York Times (Thamel, 2006), USA Today (Steeg, 2008), and the Medill Reports (Hollencamp, 2009) have published stories addressing issues of clustering in NCAA Division I men's football and basketball. Despite the different sources, the data remain the same clustering exists across NCAA Division I football and men's basketball, and the effects of clustering can reach far beyond the student-athletes and the domain of athletics. 
The New York Times reported a story about the Auburn University directed reading program that benefitted many of the football players in what appeared to be an improper way (Thamel, 2006). Directed reading courses at Auburn were designed to serve as a way for a student to delve deeper into a subject of interest than he or she could in a formal classroom setting. However, when misused, directed reading courses turned into courses that required students to complete very little work to receive an A. Through the timeframe of the NCAA investigation, it was found that Professor Thomas Petee increased his student load from 150 students to over 300 students. This dramatic increase in just one year caught the attention of the sociology department chair and led to a university-wide and NCAA investigation. This issue divided the faculty and even involved the university president. The investigation found that the sociology department became a safe haven for football student-athletes and eighteen members of the 2004 undefeated team took 97 credits directed reading courses from Professor Petee. After the New York Times story broke, Petee's directed reading courses dropped from 152 the previous fall to 25 .

Some student-athletes have applauded majors that are chosen for clustering such as Interdisciplinary Studies or University Studies because of the opportunity to take a number of classes in more than one subject. However, these majors often do not permit a student to take upper level classes in one particular subject area. Instead, they are limited to a number of lower level courses, allowing them to call their training interdisciplinary (Ganczaruk, 2004). Despite the fact that these student-athletes may have course credit in multiple disciplines, the title University Studies, Interdisciplinary Studies, or another title of the sort fails to accurately describe the student's specialty or expertise (Steeg, 2008). 
Steeg (2008) led a study and wrote an article that appeared in USA Today and concluded clustering by academic major is a dynamic that leads to negative outcomes for student-athletes. Steeg (2008) used the story of a former Kansas State University lineman who felt forced by his athletics academic adviser to major in a social science and regretted his decision to frame the narrative around clustering. The study then investigated the 2007-2008 rosters for Division I teams in football, men's basketball, women's basketball, baseball, and softball, including all 120 schools in the NCAA Division I Football Bowl Subdivision. In total, the data for analysis was comprised of 9,300 student-athletes from 654 different teams. Steeg (2008) found that $83 \%$ of the schools (118 of 142) had at least one team where clustering occurred, $34 \%$ of the teams (222 of 654$)$ had at least one cluster ( $25 \%$ or more) of student-athletes, and 125 of the teams where clustering occurred had at least $40 \%$ of the student-athletes in the same major (some analysts call this "extreme" clustering). After presenting these results, Steeg (2008) referenced comments made by former Georgia Tech Men's Basketball Head Coach Paul Hewitt to the Knight Foundation Commission about a mixed message existing about the importance of academics in college sports and then discussed how recent NCAA academic legislation such as the 40-60-80 rule and the Academic Progress Rate (APR) have created the 'perfect storm' for eligibility problems for coaches, athletic academic advisers, and student-athletes. Despite the fact that this study was not published in a peer-reviewed journal and the data was limited to one sporting season, the results provide a negatively implicating snapshot of the effects of clustering.

The Associated Press released an article in 2011 published on the NCAA's website entitled, "Athletes sticking together in classes." The subtitle of the article read, "More than half of BCS schools have players clustered in majors.” The author framed the student-athlete's decision to pursue a clustered major at his or her institution as a conscious decision, but also 
mentions the potential impact of the NCAA APR. NCAA vice president of academic and membership affairs Kevin Lennon said, "Those [Academic majors] are personal decisions that every student-athlete has to make, just like any student." Lennon also stated, "We need to remind ourselves that before the reform effort, some students weren't getting a degree at all...The APR is incredibly significant. We have more young people moving toward a degree. We have many more getting degrees. That's the most important thing."

\section{Recent Studies of Clustering}

Fountain and Finley (2009) investigated academic clustering in the Atlantic Coast Conference (ACC) among football players. The ACC is comprised of twelve teams, but data were available for eleven. The researchers explored whether clustering was different between white and minority (non-white) players and if there were certain majors that were targeted for academic clusters. They utilized Case et al.'s (1987) operational definition of clustering and limited their analysis to upperclassmen (juniors and seniors) because underclassmen were either more likely to have an undeclared major or a general studies major until they officially declared a major after their sophomore year. The data set was comprised of 394 student-athletes, with $41.4 \%$ of the student-athletes identified as White and $58.6 \%$ of the student-athletes identified as minority. The researchers were limited to identifying the student-athletes as white or minority because of the limitation of using images from the Internet or printed media guide. To investigate correlations between the student-athletes' majors and their race, Fountain and Finley (2009) classified each major into one of nine broad areas of study and utilized chi-squared tests.

Fountain and Finley (2009) investigated three research questions: 1) Does academic clustering occur among ACC football players? 2) Is there a difference in prevalence of clustering when considering White and Minority subgroups? 3) Are there multiple majors at these schools 
that also exceed the threshold to be considered clustered? All eleven schools showed evidence of clustering, showed clustering happened more frequently for minority players than white players, and that there were distinct majors that were subject to clustering dependent on the institution (Fountain and Finley, 2009). The researchers also found that some institutions had more than one major with a cluster of student-athletes. Fountain and Finley (2009) indicated that future research should address whether the NCAA's recent academic legislation led to a change in clustering and should address how players select their majors.

Schneider, Ross, and Fisher (2010) also conducted a recent study to examine clustering by academic major in three years $(1996,2001,2006)$ in a ten-year period in the Big 12 conference. The ten-year period allowed longitudinal analysis and opened the possibility of identifying patterns and trends in clustering over time. The researchers chose the Big 12 conference because each member institution holds a strong reputation for academic and athletic quality in the Midwest. Schneider, Ross, \& Fisher (2010) also limited the number of eligible student-athletes for the study to juniors and seniors. Schneider, Ross, and Fisher (2010) found that clustering was present at many of the Big 12 member institutions in each of the three years included in the study. As Case et al. (1987) found, the specific clustered majors varied by institution. The researchers did not classify the majors by area of study, instead they reported the data from each institution that exhibited clustering.

Fountain \& Finley (2011) conducted a longitudinal analysis of student-athlete majors in a "highly competitive" Division I football program. This study was the first of its kind, and investigated the rate that athletes transfer in and out of academic majors, particularly transferring into majors with clusters of athletes. The researchers posited five research questions: 1) Did clustering occur over time? If so, was it different for white and minority players? 2) What was 
the common academic progression for students who started in general education (University Studies)? 3) Were players more likely to migrate into an academic cluster if they received "star" ranking from Scout.com during their senior year in high school? 4) Were players who were drafted into the National Football League (NFL) likely to have been enrolled in a clustered major? 5) Were there academic programs that players migrated away from during their academic careers?

Fountain \& Finley (2011) found that many student-athletes had a variety of majors in their first two years, but tended to move into a group of similar majors in their last two years. Although the researchers did not state any possible reasons for the shift, research indicates that the shift may have occurred to ensure that eligibility was maintained. The movement into similar majors was a movement by cluster. Again, this dynamic affected all football players, but disproportionately affected minority players. A new finding in this study suggests that clustering is a systematic process, as the findings indicated that players who were highly rated before entering college and players who left college and were drafted into the National Football League (NFL) appeared to cluster in the same major.

It is clear that clustering occurs in intercollegiate sports, particularly revenue sports, and the implications are apparent. However, there has been little research to document the effects of academic clustering on the individuals most affected by its practice - the student-athletes. There has also been no published research that examines clustering by academic major from the student-athlete perspective.

\section{Implications of Clustering}

Researchers have performed several studies investigating clustering in male revenuegenerating sports for years and proven its existence (Case et al., 1987; Fountain \& Finley, 2009, 
2011; Schneider, Ross, Fisher, 2010; Steeg, 2008), yet clustering seems to have magnified since those times with the investment of third party organizations as sponsors of college sports (Sharp $\&$ Sheilley, 2008). These corporations have undoubtedly brought large amounts of money to the sport participation, resulting in participating institutions receiving more money for their athletic departments, coaches, and services available to players. However, the downside of this increased investment is more time required for student-athletes for practices, travel for competitions, and other outside of the classroom obligations.

Despite the seemingly negative implications of clustering by student-athletes, NCAA President Myles Brand has publicly supported academic clustering (Hollencamp, 2009). Others have emphasized the importance of accurately of reporting clustering (Elfman, 2009; Otto, 2012). At the University of Maryland, the largest major is criminology and criminal justice for all undergraduate students. However, the major ranks second among student-athletes. Dr. Charles Wellford, a criminology and criminal justice professor at Maryland stated that it's important to compare student-athlete majors to the majors of the general student population (Elfman, 2009). Otto (2012) echoed Wellford's sentiments when she emphasized the importance of comparing student-athlete majors to the majors of the general student population. Fountain and Finley's 2009 and 2011 studies did not compare the majors of the student-athletes to the rest of the college's student body.

Otto (2012) conducted a study of clustering that was designed to improve the accuracy of previous studies of clustering. Otto (2012) referenced Case et al.'s (1987) seminal study and emphasized the importance of differentiating between 'academic major' and 'area of study'. Otto (2012) also stressed the importance of comparing the student-athletes' majors to the majors of the general student body and posed that it is not enough to solely analyze the majors of student- 
athletes alone. The comparison component of analysis is crucial to accurately identifying clustering by academic major because it is the way that Case et al. (1987) initially defined clustering by academic major. Otto (2012) investigated clustering amongst upperclassmen football players in the Pac-10 for the 2009-2010 season $(n=415)$. The Pac-10 was chosen because it is a BCS Conference that had not been previously studied. Otto (2012) found that clustering by academic major was present in seven of the ten Pac-10 programs, extreme clustering (40\% or more) was present in one of the seven programs, and that clustering occurred in two separate majors in one of the seven programs. Otto (2012) also found that there was a significant difference in the presence of clustering by academic major when the results are reported based on an 'area of study' instead of a 'major.' In this study an average of $30.5 \%$ of student-athletes clustered when results were reported by 'major' jumped to an average of $78 \%$ when the results were reported by 'area of study'.

Clustering also has strong implications about the current state of minority student-athlete as compared to white student-athletes. The migration of minority student-athletes into college sports was a process with prevalent exploitation in the middle of the $20^{\text {th }}$ century. However, current research on academic clustering and student-athletes after they leave college indicates that the NCAA may not have sufficiently addressed athlete exploitation as academic legislation reform and improved graduation rates seem to indicate. It is hard to empirically research this topic because there is little data that depict clustering itself as a negative phenomenon.

Sanders and Hildenbrand (2010) investigated some potential causes of clustering by academic major and whether clustering by academic major leads to future income inequalities between student-athletes and non-athlete students. The scholars developed six hypotheses to address the question of why student-athletes cluster. The first hypothesis, the selection 
hypothesis, argued that student-athletes may be predisposed select their majors due to their often lower entering skills, less academic college preparation, and different preexisting interests than non-athlete students. The second hypothesis, the structure hypothesis, argued that the structure and demands of collegiate athletics forces student-athletes to choose majors that allow them the most time to commit to their sport and fulfill their role as student-athlete. Thus, when given the choice between social sciences majors and other academic majors, the researchers posed that student-athletes would be more likely to select social sciences majors than non-athlete students. The third hypothesis, the gender hypothesis, argued that male student-athletes would be more likely than female student-athletes to choose a social sciences major. The fourth hypothesis, the race hypothesis, argued that African-American student-athletes would be more likely to choose a social science major than student-athletes of any other race. The fifth hypothesis, the high-profile sport hypothesis, argued that high-profile student-athletes would be more likely to choose a social science major than student-athletes that do not participate in high-profile sports (football men's basketball, women's basketball). The sixth hypothesis, the diminished income hypothesis, argued that student-athletes would have lower projected incomes after college than non-athlete students because of the large number of student-athletes in social science majors. Sanders and Hildenbrand (2010) analyzed data from a database of students from a Midwestern land grant university. The database included 12,402 students $(n=12,402)$ with records of fourteen semesters for each student. The researchers separated student-athletes from non-athlete students and multinomial logistic regressions to compare the variables such as first major, final major, athlete, male athlete, African American athlete, and high-profile athlete. Sanders and Hildenbrand (2010) found that clustering by academic major occurred as a result of the selection hypothesis and the structure hypothesis, clustering by academic major is most prevalent among African-American 
student-athletes, clustering also affects male student-athletes and student-athletes who play bigtime sports, and clustering does not affect female student-athletes, student-athletes who are not African-American, and student-athletes who do not play high-profile sports. Finally, the researchers found that student-athlete projected incomes are only temporarily lower than nonathlete student projected incomes. Sanders and Hildenbrand's (2010) study was limited by the use of just one institution, but made a strong empirical argument for the negative outcomes of clustering by academic major.

\section{NCAA GOALS and SCORE Studies}

The NCAA conducted research on the student-athlete academic experience, particularly the paths that student-athletes follow in pursuing a major. Two studies are at the forefront of this research, Growth, Opportunities, Aspirations, and Learning of Students in college (GOALS) and Study of College Outcomes and Recent Experiences (SCORE). The GOALS study examines current student-athletes about their experiences and the SCORE study examines former studentathletes. The NCAA uses the following pneumonic to help remember the purposes of the studies: set GOALS in college, check on the SCORE later (NCAA, 2008).

The GOALS questionnaire is divided into six parts: College Athletic Experience, College Academic Experience, College Social Experience, The Student-Athlete Experience, Health and Wellbeing, Time Commitments, Background Information, and Additional Feedback and Comments. Questions in the College Athletic Experience section ask about academic and athletic expectations prior to coming to college and the likelihood of becoming a professional or Olympic athlete in their current sport. Questions in the College Academic Experience section asks about student-athletes' major area of study, primary reason for selecting a major, and feelings about choice of major, and the chances of being involved in opportunities such as study 
abroad, internships, independent study courses, and a culminating academic experience project such as a thesis. Questions in the College Social Experience section ask about involvement in extracurricular activities, community service, and the team social environment. Each of these questions provide further insight into the day-to-day experiences of student-athletes inside and outside of the arena of sport.

\section{Summary}

Intercollegiate athletics have been a topic of research across many academic disciplines, but there is a dearth of research about the student-athletes that participate outside of the NCAA Division I classification and student-athletes who compete for HBCUs. Despite the lacking research, the fact remains that there are more student-athletes that compete outside of NCAA Division I than compete in the division. Academic legislation affects student-athletes across NCAA Divisions, but the change in academic legislation has occurred most often for NCAA Division I student-athletes.

NCAA academic legislation for Division I student-athletes has shifted its focus from precollege eligibility with propositions 16 and 48 to real-time academic progress monitoring with the APR. This shift has placed more of an emphasis on what happens semester by semester for student-athletes instead of emphasizing their preparation for college. Harsh penalties for studentathletes, teams, and athletic departments as a whole may influence the information that studentathletes receive about pursuing a meaningful education versus maintaining eligibility to compete. The APR system only exists in NCAA Division I. NCAA Division II institutions are governed by the Academic Success Rate (ASR), which does not have the same implications as the APR, yet still holds these institutions to an academic standard. In APR and ASR ratings, student- 
athletes in football and men's basketball consistently rank lower than student-athletes in other sports and black student-athletes consistently rank lower than white student-athletes.

Although HBCUs were opened with a unique educational mission, student-athletes that participate at these institutions face similar challenges as student-athletes who compete at other types of institutions. However, the student-athletes may not receive the same support that student-athletes at other institutions receive because of financial concerns that uniquely affect HBCUs. The NCAA has sought to gain insight to the student-athlete academic experience during and after their time in school, but this data is laden with descriptive themes that do not provide in-depth information about the daily hassles and major decisions that student-athletes make in their academic and athletic lives.

Clustering by academic major has been empirically researched since 1987. Some of the research about clustering suggests that it is a form of exploitation of student-athletes who pursue clustered majors (Steeg, 2008; Hollencamp, 2009) while other research about clustering simply draws attention to a dynamic that appears to uniquely occurred within the student-athlete population (Sanders \& Hildenbrand, 2010; Otto, 2012) and occurred disproportionately for minority student-athletes as compared to white student-athletes. Research about clustering is significant because a student's academic major can influence his or her first job and career trajectory. Student-athletes receive input from their families, coaches, athletic academic advisors, and other individuals that may urge them to make considerations about their athletic competition and eligibility to compete that non-athlete students do not have. 


\section{References}

Adler, P., \& Adler, P. (1985). From idealism to pragmatic detachment: The academic performance of college athletes. Sociology of Education, 58, 241-250. DOI: $10.2307 / 2112226$

Allen, W. R., Jewell, J. O., Griffin, K. A., Wolf, D. S. (2007). Historically black colleges and universities: Honoring the past, engaging the present, touching the future. The Journal of Negro Education, 76(3), 263-280.

Associated Press. (8 September 2011). Athletes sticking together in classes: More than half of BCS schools have players clustered in majors. NCAA.com. Retrieved from http://www.ncaa.com/news/football/article/2011-09-05/athletes-sticking-together-classes

Baucom, C., \& Lantz, C. D. (2001). Faculty attitudes toward male division ii student-athletes. Journal of Sport Behavior, 24(3), 265-277.

Bettez, S. C., \& Suggs, V. L. (2012). Centering the educational and social significance of hbcus: A focus on the educational journeys and thoughts of african american scholars. Urban Review, 44, 303-310. doi: 10.1007/s11256-012-0201-x

Blackman, P. (2008). The NCAA's academic performance program: Academic reform or academic racism? UCLA Entertainment Law Review, 15(2), 225-289.

Brooks, D., Etzel, E., Ostrow, A. (1987). Job responsibilities and backgrounds of NCAA division I athletic advisors and counselors. The Sport Psychologist, 1, 200-207.

Brown II, M. C. (2013). The declining significance of historically black colleges and universities: Relevance, reputation, and reality in obamamerica. Journal of Negro Education, 82(1), 3-19.

Cantey, N. I., Bland, R., Mack, L. R., \& Joy-Davis, D. (2013). Historically black colleges and 
universities: Sustaining a culture of excellence in the twenty-first century. Journal of African American Studies, 17, 142-153. doi: 10.1007/s12111-011-9191-0

Case, B., Greer, S., \& Brown, J. (1987). Academic clustering in athletics: Myth or reality? Arena Review, 11(2), pp. 48-56.

Charlton, R. (2011). The role of policy, rituals and language in shaping an academically focused culture in HBCU athletics. Journal of Issues in Intercollegiate Athletics, 4, 120-148.

Chu, D. (1985). The american conception of higher education and the formal incorporation of intercollegiate sport. In D. Chu, J.O. Seagrave, \& B.J. Becker (Editors), Sport in higher education (35-55). Champaign, IL. Human Kinetics Publishers, Inc.

Coakley, J. (2004). Sports in Society: Issues \& Controversies, Eighth Edition. New York, NY, McGraw-Hill Higher Education.

Coakley, J. (2009). Sports in Society: Issues \& Controversies, Tenth Edition. New York, NY, McGraw-Hill Higher Education.

Cooper, J. N., Hawkins, B. (2012). A place of opportunity: Black male student athletes' experiences at a historically black university. Journal of Intercollegiate Sport, 5, 170-188.

Coupet, J. (2013). Historically black colleges and universities and resource dependence: A Chow test of production functions. Journal of Higher Education Policy and Management, 35(4), 355-369. Doi: 10.1080/1360080X.2013.812054

Cunningham, G. B. (2012). Diversity issues in academic reform. Journal of Intercollegiate Athletics, 5, 54-59.

Davenport, J. (1985). From crew to commercialism. In D. Chu, J.O. Seagrave, \& B.J. Becker (Editors), Sport in higher education (5-15). Champaign, IL. Human Kinetics Publishers, Inc. 
Davis, T. (1996). The myth of the superspade: The persistence of racism in college athletics. Fordham Urban Law Journal, 22(3), 614-698.

Davis, T. (1996). African-american student-athletes: Marginalizing the ncaa regulatory structure?, Marquette Sports Law Review, 6(2), 199-227.

Dent, M., Sanserino, M., \& Werner, S. (2014). Do colleges drop the ball with student-athletes?

Drezner, J. A., Rogers, K. J., \& Horneff, J. G. (2011). Automated external defibrillator use at NCAA division ii and iii universities. British Journal of Sports Medicine, 45, 1174-1178. doi:10.1136/bjsm.2009.070052

Dubois, P.E. (1985). The occupational attainment of former college athletes: A comparative study. In D. Chu, J.O. Seagrave, \& B.J. Becker (Editors), Sport in higher education (235248). Champaign, IL. Human Kinetics Publishers, Inc.

Edwards, H. (1985). Educating black athletes. In D. Chu, J.O. Seagrave, \& B.J. Becker (Editors), Sport in higher education (373-384). Champaign, IL. Human Kinetics Publishers, Inc.

Elfman, L. (2009, February 23). Are minority football players being pushed into pointless majors? Diverse. Retrieved from http://diverseeducation.com/article/12325/

Ellis, L., \& Wilson, R. (2014, January 6). Professors in class on time? Check. The Chronicle of Higher Education.

Engstrom, C., Sedlacek, W, \& McEwan, M. (1995). Faculty attitudes toward male revenue and non-revenue student-athletes. Journal of College Student Development, 36, 217-227.

Esters, L. L., \& Strayhorn, T. L. (2013). Demystifying the contributions of public land-grant historically black colleges and universities: Voices of HCU presidents, The Negro Educational Review, 64 (1-4), 119-134. 
Exkano, J. (2013). Toward an African cosmology: Reframing how we think about historically black colleges and universities. Journal of Black Studies, 44(1), 63-80. doi: $10.1177 / 0021934712465313$

Fester, R., Gasman, M., Nguyen, T. (2012). We know very little: Accreditation and historically black colleges and universities. Journal of Black Studies, 43(7), 806-819. doi: $10.1177 / 0021934712453467$

Fields, S. K. (2012). Are we asking the right questions?: A response to the academic reforms research the NCAA. Journal of Intercollegiate Sport, 5, 60-64.

Fountain, J. J., and Finley, P. S. (2009). Academic majors of upperclassmen football players in the atlantic coast conference: An analysis of academic clustering comparing white and minority players. Journal of Issues in Intercollegiate Athletics, 2, 1-13.

Fountain, J. J., and Finley, P. S. (2011). Academic clustering: A longitudinal analysis of a division I football program. Journal of Issues in Intercollegiate Athletics, 4, 24-41.

Galotti, K. M. (1999). Making a "major" real-life decision: College students choosing and academic major. Journal of Educational Psychology, 91(2), 379-387.

Galotti, K. M., Ciner, E., Altenbaumer, H. E., Geerts, H. J., Rupp, A., Woulfe, J. (2006). Decision-making styles in a real-life decision: Choosing a college major. Personality and Individual Differences, 41, 629-639. doi:10.1016/j.paid.2006.03.003

Ganczaruk, S. (2004). Student athletes gravitate toward similar degrees. Devil's Tale. Retrieved May 13, 2007 from http://cronkitezine.asu.edu/spring2004/athletes.html

Gaston-Gayles, J. L. (2003). Advising student athletes: An examination of academic support programs with high graduation rates. NACADA Journal, 23(1\&2), 50-57. doi: $10.12930 / 0271-9517-23.1-2.50$ 
Gaston-Gayles, J. L. (2004). Examining academic and athletic motivation among Student athletes at a Division I university. Journal of College Student Development, 45(1), 75-83. DOI: $10.1353 /$ csd.2004.0005

Gruber, C. A. (2003). What every academic advisor should know about advising student athletes. NACADA Journal, 23(1\&2), 44-49. doi: 10.12930/0271-9517-23.1-2.44

Hanford, G. H. (1985). Proposition 48. In D. Chu, J.O. Seagrave, \& B.J. Becker (Editors), Sport in higher education (373-384). Champaign, IL. Human Kinetics Publishers, Inc.

Hodge, S. R., Bennett III, R. A., \& Collins, F. G. (2013). Historically black colleges and universities' athletes and sport programs: Historical overview, evaluations, and affiliations. In D. Brooks and R. Althouse (Editors), Racism in college athletics, $3^{\text {rd }}$ edition (63-104). Morgantown, WV: FiT Publishing Company.

Hodge, S. R., Bennett III, R. A., \& Collins, F. G. (2013). The journey of the black athlete on the HBCU playing field. In D. Brooks and R. Althouse (Editors), Racism in college athletics, $3^{\text {rd }}$ edition (105-133). Morgantown, WV: FiT Publishing Company.

Hollencamp, K. (2009, February 23). NCAA academic ratings may force students to choose between dreams. Medill Reports. Retrieved from http://news.medill.northwestern.edu/Chicago/news.aspx?id=118185\&print $=1$

Johnson, J. E., Wessel, R. D., Pierce, D. A. (2012). The influence of selected variables on NCAA academic progress rate. Journal of Issues in Intercollegiate Athletics, 5, 149-171.

Kim, M.M., \& Conrad, C.F. (2006). The impact of historically black colleges and universities on the academic success of african-american students. Research in Higher Education, 47(4), 399-427. Doi: 10.1007/s11162-005-9001-4

Judge, L.W., Bell, R. J., Theodore, R., Simon, L., \& Bellar, D. (2012). An exploratory 
examination of burnout in NCAA division ii athletes. Journal of Intercollegiate Sport, 5, 230-240.

Leach, J. K., \& Patall, E. A. (2013). Maximizing and counterfactual thinking in academic major decision making. Journal of Career Assessment, 21(3), 414-429. doi:

$10.1177 / 1069072712475178$

Melendez, M. C. (2010). Psychosocial influences on college adjustment in division 1 studentathletes: The role of athletic identity. Journal of College Student Retention, 11(3), 345361.

Montmarquette, C., Cannings, K, Mahseredjian, S. (2002). How do young people choose college majors? Economics of Education Review, 21, 543-556.

doi: $10.1016 / \mathrm{S} 0272-7757(01) 00054-1$

Morgan, W. J. (2012). The academic reform of intercollegiate athletics: The good, the problematic, and the truly worrisome. Journal of Intercollegiate Sport, 5, 90-97.

NCAA. (2011a). Differences among the three divisions: Division I. Retrieved December 16, 2013

from

http://www.ncaa.org/wps/wcm/connect/public/ncaa/about+the+ncaa+old/who+we+are/di fferences + among + the + divisions/division $+\mathrm{i} /$ about + division $+\mathrm{i}$

NCAA. (2011b). Differences among the three divisions: Division II. Retrieved December 16, 2013 from http://www.ncaa.org/wps/wcm/connect/public/ncaa/about+the+ncaa+old/who+we+are/di $\underline{\text { fferences }+ \text { among }+ \text { the }+ \text { divisions/division }+\mathrm{ii} / \text { about }+ \text { division }+\mathrm{ii}}$

NCAA. (2011c). Division II elevator speech. Retrieved December 16, 2013 from 
http://www.diicommunity.org/uploads/documents/PDF\%20Why\%20Choose\%20Division \%20II 98623.pdf

NCAA. (2013a). Welcome to Division III. Retrieved December 16, 2013 from http://www.ncaa.org/wps/wcm/connect/public/NCAA/Division+III/About+Division+III

Nichols, J. C. (2004). Unique characteristics, leadership styles, and management of historically black colleges and universities. Innovative Higher Education, 28(3), 219-229.

Doi: 10.1023/B:IHIE.0000015109.49156.fb

Nite, C. (2012). Challenges for supporting student-athlete development: Perspectives from an NCAA division ii athletic department, Journal of Issues in Intercollegiate Athletics, 5, 114.

Nyquist, E.B. (1985). The immorality of big-power intercollegiate athletics. In D. Chu, J.O. Seagrave, \& B.J. Becker (Editors), Sport in higher education (101-114). Champaign, IL. Human Kinetics Publishers, Inc.

Otto, K. (2012). Demonstrating the importance of accuracy in reporting results of academic clustering. Journal for the Study of Sports and Athletes in Education, 3, 293-310. http://dx.doi.org/10.1179/ssa.2012.6.3.293

Palmer, R. T., Davis, R. J., \& Maramba, D. C. (2010). Role of an HBCU in supporting academic success for underprepared black males. The Negro Educational Review, 61(1-4), 85-106).

Paskus, T. S. (2012). A summary and commentary on the quantitative results of current NCAA academic reforms. Journal of Intercollegiate Sport, 5, 41-53.

Petr, T. A.,\& McArdle, J.J. (2012). Academic research and reform: A history of the empirical basis for NCAA academic policy. Journal of Intercollegiate Sport, 5, 27-40.

Purdy, D. A., Eitzen, D. S. \& Hufnagel, R. (1982). Are athletes also students? The educational 
attainment of college athletes. Social Problems, 29, 439-448. DOI: 10.2307/800032

Redd, K. E. (1998). Historically black colleges and universities: Making a comeback. New Directions for Higher Education, 102, 33-43. DOI: 10.1002/he.10203

Renick, J. (1974). The use and misuse of college athletics. The Journal of Higher Education, 45(7), 545-552. DOI: $10.2307 / 1980793$

Roach, R. (2004). Academics and athletics: Playing for the same team. Black Issues in Higher Education, 21(4), 26-31.

Sack, A. (1986). Jan Kemp wins one for integrity. Journal of Sport and Social Issues, 10(1), 3-5. doi: $10.1177 / 019372358601000101$

Sanders, J. P., \& Hildenbrand, K. (2010). Major concerns? A longitudinal analysis of studentathletes' academic majors in comparative perspective. Journal of Intercollegiate Sport, 3, 213-233.

Schneider, R. G., Ross, S. R., \& Fisher, M. (2010). Academic clustering and major selection of intercollegiate student-athletes. College Student Journal, 44(1), 64-70.

Sellers, R. M., Chavous, T. M., \& Brown, T, N. (2002). Uneven playing field: The impact of structural barriers on the initial eligibility of African American student-athletes. Paradoxes of Youth and Sport, 173-186.

Sharp, L. A., \& Sheilley, H. K. (2008). The insitution's obligations to athletes. New Directions for Higher Education, 142, 103-113.

Shropshire, K. L. (2013). Complexity and memory: Financial related issues among hbcus. Journal of Intercollegiate Sport, 6, 76-78.

Simons, H. D., Bosworth, C., Fujita, S., \& Jensen, M. (2007). The athlete stigma in higher education. College Student Journal, 41(2), 251-273. 
Sojka, G.S. (1985). The evolution of the student-athlete in america: From the divinity to the divine. In D. Chu, J.O. Seagrave, \& B.J. Becker (Editors), Sport in higher education (1733). Champaign, IL. Human Kinetics Publishers, Inc.

Steeg, J. (2008). UNLV athletes question degrees in university studies. USA Today. Retrieved from http://www.usatoday.com/sports/college/2008-11-19-unlv-university-studiesdegree N.htm

Steeg, J.L., Upton, J., Bohn, P., Berkowitz, S. (2008). USA Today. Retrieved from http://usatoday30.usatoday.com/sports/college/2008-11-18-majors-cover_N.htm

Stevenson, J. M. (2007). From founding purpose to future positioning: Why historically black colleges and universities must maintain but modify mission, Jackson State University Researcher, 21(3), 99-102.

Stuart, R. (2012). HBCUs could be hit hard by new NCAA rules. Diverse Education. Retrieved from http://diverseeducation.com/article/17098/\#

Suggs, W. (2003). Jock majors: Many colleges allow football players to take the easy way out. The Chronicle of Higher Education, 49(17), 33. Retrieved from http://chronicle.com/article/Jock-Majors/32843

Thamel, P. (2006). Top grades and no class time for auburn players. The New York Times. Retrieved from http://www.nytimes.com/2006/07/14/sports/ncaafootball/14auburn.html?pagewanted=all

Wessel, J. L., Ryan, A. M., Oswald, F. L. (2008). The relationship between objective and perceived fit with academic major, adaptability, and major-related outcomes. Journal of Vocational Behavior, 72, 363-376. Doi: http://dx.doi.org/10.1016/j.jvb.2007.11.003 
Wolniak, G. C., Seifert, T. A., Reed, E. J., Pascarella, E. T. (2008). College majors and social mobility. Research in Social Stratification and Mobility, 26, 123-139. Doi: http://dx.doi.org/10.1016/j.rssm.2008.02.002 


\section{Appendix $C$}

\section{NCAA GOALS Sorting Sheet}

Classify each academic major under a particular area of study. Areas of study are listed below.

1. Biological Sciences (Zoology, Physiology, etc.)

2. Business (Accounting, Marketing, Personnel, etc.)

3. Communications (Journalism, Public Relations, etc.)

4. Education (Elementary, Special, etc.)

5. Engineering, Computer/Information Sciences

6. Exercise, Sports, Kinesiology

7. Humanities and Fine Arts (Music, Religion, English, etc.)

8. Physical Sciences and Mathematics (Chemistry, etc.)

9. Professional Studies (Nursing, Occupational Therapy, etc.)

10. Social Sciences (Psychology, History, Economics, etc.)

11. Other Academic Field 


\section{University 1}

- Accounting
- Business Management
- Chemistry
- Community Health
- Criminal Justice
- Finance
- Forensic Biology
- Integrated Studies
- Management
- Management Information Systems
- Marketing
- Mass Communications
- Movement Science
- Physical Education
- Physical Therapy
- Physics
- Pre-Education/Elementary Education
- Psychology
- Secondary Education
- Social Work
- Special Education
- Sport Management
- Sport Science

\section{University 2}

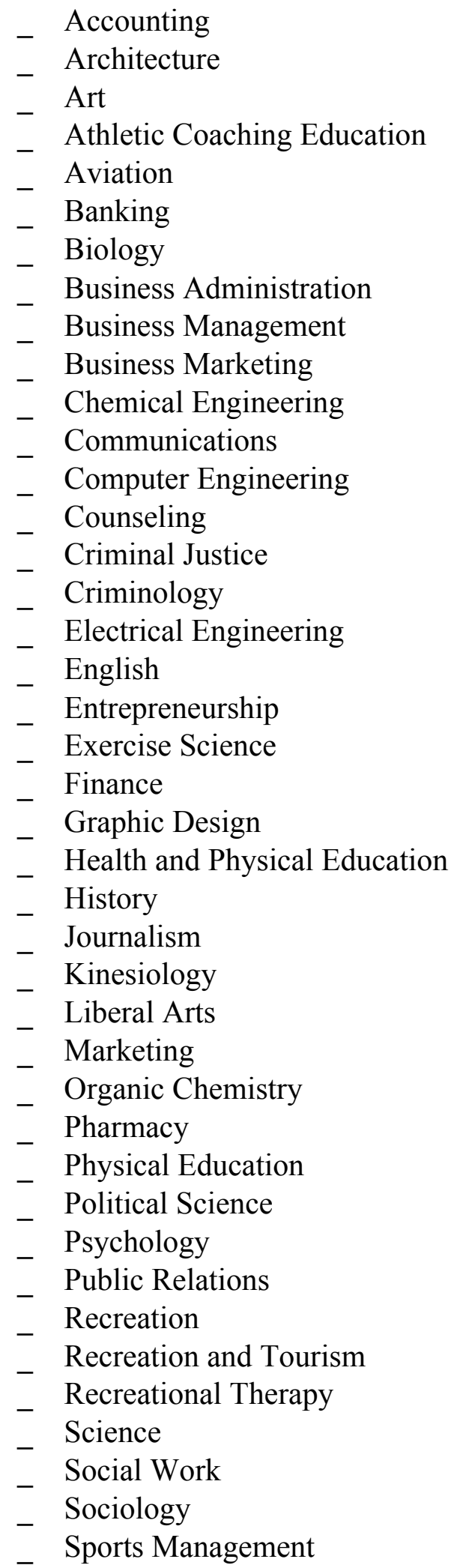




\section{University 3}

- Accounting
- Biology
- Business
- Business Administration
- Business Administration-
Marketing
- Computer Science
- Criminal Justice
- English
- Exercise Science
- General Studies
- Human Ecology
- Social Work
- Sociology

\section{University 4}

_ Accounting

- Biology

_ Broadcast Journalism

- Business

- Business Administration

_ Business Marketing

- Business Management

_ Business Law

- Communications

- Computer Engineering

- Criminal Justice

- Education

_ Electrical Engineering

_ English

_ Industrial Engineering

_ Information Systems

_ Marketing

- Mathematics

- Medical Technology

_ Physical Education

- Psychology

_ Public Relations

- Sociology

- Speech Communications

- Sports Administration

_ Telecommunication 


\section{University 5}

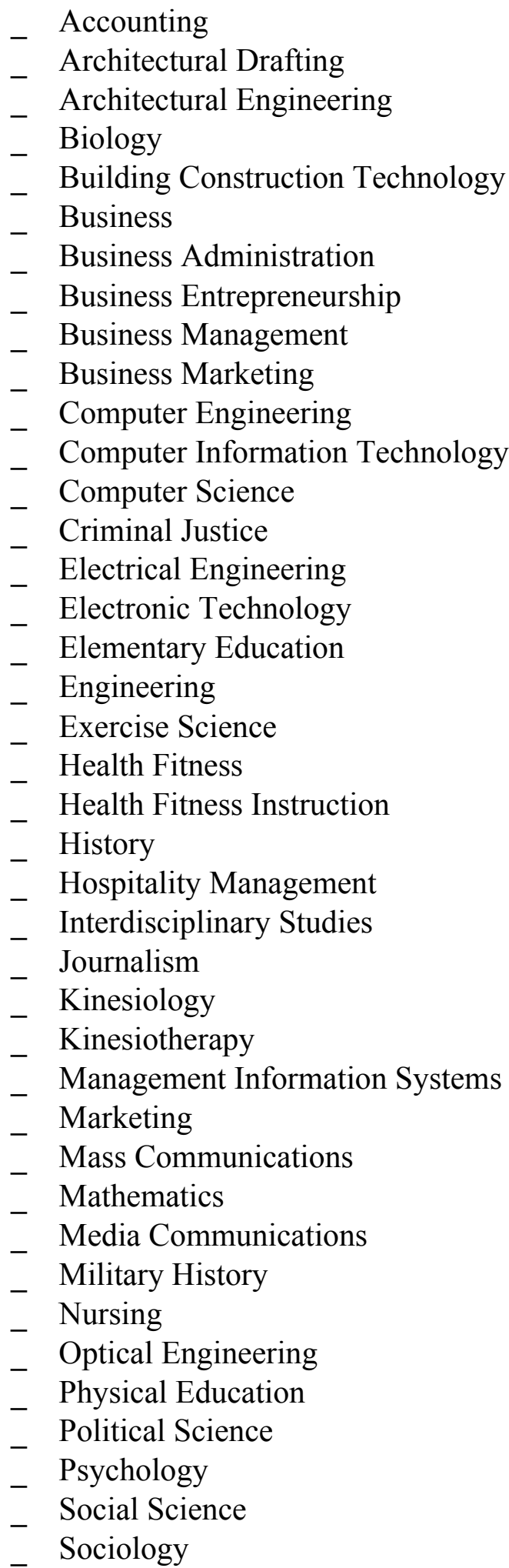

\section{University 6}

_ Accounting

- Animal Science

_ Applied Engineering Technology

- Business Management

- Chemical Engineering

_ Computer Science

- Construction Management

- Criminal Justice

- Electrical Engineering

- Electronics Technology

_ Engineering

- English

- Finance

_ Graphic Communication Systems

- History Education

- Information Technology

_ Interdisciplinary General English

- Journalism and Mass

- Communications

_ Liberal Studies (Pre Law)

- Management Information Systems

- Mathematics

_ Political Science

- Psychology

- Social Work

- Sport Science and Fitness Management 


\section{University 7}

_ Accounting

- Art

_ Athletic Training

- Biology

_ Broadcast Journalism

- Business

- Business Management

- Chemistry

- Communications

- Computer Information Systems

- Computer Science

- Criminal Justice

- Education

- English

- Environmental Science

- Exercise Sports Science

- Finance

- Fitness and Wellness

- Health

- Health Education

- History

_ Hospitality and Tourism

- Journalism

- Kinesiology

- Law (J.D.)

- Marketing

_ Mass Communications

- Math

- Nursing

- Parks and Recreation Management

- Pharmaceutical Science

- Physical Education

_ Physical Therapy

- Physics

- Political Science

- Pre-Med

- Psychology

- Social Science

- Social Work

- Sociology

_ Sports Management

\section{University 8}

_ Accounting

- Behavior Analysis

- Biology

- Business Administration

_ Business Management

_ Business Marketing

- Chemistry

_ Civil Engineering

- Computer Engineering

- Computer Information Systems

- Criminal Justice

- Electrical Engineering

- Mass Communications

- Mathematics

- Mechanical Engineering

_ Political Science 


\section{University 9}

_ Biology

- Business Economics

- Business Management

- Business Marketing

- Chemistry

- Child Development

- Civil Engineering Technology

- Criminal Justice

- Early Childhood Education

- Education

- Elementary Education

- Family and Consumer Science

- Health Sciences

- History

- Industrial Engineering Technology

- Industrial Technology

- Mass Communication

- Mathematics Education

- Mechanical Engineering Technology

_ Nursing

- Physical Education

- Political Science

- Sociology

- Sport Communication

- Sport Management

_ Sport Medicine

- Technology Education

- Theater

\section{University 10}

_ Accounting

_ Banking and Finance

_ Biology

- Broadcast Journalism

- Business

_ Business Administration

- Business Information Systems

_ Business Management

- Communications

- Computer Graphics

- Computer Technology

- Criminal Justice

- Early Childhood Education

- Education

- Engineering

_ English

_ Graphic Design

_ History

_ Marketing

- Mathematics

- Mathematics Education

- Music

- Music Technology

_ Nursing

- Pedology

- Political Science

- Psychology

_ Public Administration (M.A.)

_ Secondary Education

- Social Work

_ Sociology (Criminal Justice)

- Sports Management

- Theater Arts

_ Visual Arts 


\section{University 11}

- Accounting
- Art
- Aviation Science
- Biology
- Business
- Business Administration
- Business Management
- Business/Accounting
- Chemistry
- Communications
- Computer Science
- Criminal Justice
- Education
- Elementary Education
- Engineering
- Engineering Technology
- English
- General Studies
- Graphic Design
- History
- Industrial Technology
- Marketing
- Mathematics
- Pharmacy
- Physical Education
- Physical Education - Aquatic and
- Fitness
- Physical Therapy
- Political Science
- Pre-Medicine
- Sociology
- Sport Science
- Management
- Medicine
-

\section{University 12}

- Biology

_ Business Administration

_ Business Management

- Criminal Justice

Education

_ Elementary Education

- English

_ Entrepreneurship

- Finance

- Fire Science

- Forensic Science

- Geography

- Geology

- Graphic Design

- Marketing

- Mass Communications

- Physical Education

- Pre-Law

- Pre-Med

_ Pre-Nursing

- Psychology

- Sociology

_ Sports Science 


\section{University 13}

- Accounting
- Biology
- Business Administration
- Business Management
- Chemistry
- Communication Arts
- Communications
- Community Health
- Computer Engineering
- Computer Information Engineering
- Computer Information Systems
- Computer Science
- Criminal Justice
- Criminology
- Education
- Elementary Education
- Engineering
- English
- Graphic Arts
- Health Education
- History
- Information Systems Engineering
- International Business
- Marketing
- Mathematics
- Music Business Technology
- Physical Education
- Secondary Education
- Sports Management
- Visual Arts
- Visual Performing Arts
-

\section{University 14}

- Accounting

- Anthropology

_ Biochemistry

- Biology

Broadcast Journalism

- Business

- Business Administration

_ Business Management

_ Business Marketing

- Computer Science

- Criminal Justice

- Education

- Engineering

_ English

- Environmental Science

- Finance and Management

- Health Science

- History

_ History Education

_ Information Technology

Journalism

- Management

- Mass Communications

- Physical Education

_ Physical Therapy

- Physics

- Political Science

- Pre-Engineering

- Sociology

- Sport Management

_ Visual Arts 


\section{University 15}

- Accounting
- Art
- Athletic Training
- Biology
- Business
- Business Administration
- Chemistry
- Civil Engineering
- Communications
- Computer Information Systems
- Criminal Justice
- Design/Graphic Art
- Education
- Electrical Engineering
- Elementary Education
- Engineering-Math
- Forensic Science
- General Studies
- Human Performance \& Wellness
- Liberal Studies
- Mass Communication
- Mathematics
- Mechanical Engineering
- Music
- Physical Education
- Physical Science
- Political Science
- Psychology
- Sociology
- Visual Arts
-

\section{University 16}

_ Accounting

- Agriculture

- Animal Science

- Biology

_ Business Management

_ Business Marketing

- Computer Engineering

- Computer Information Systems

- Computer Science

- Counselor Education

- Criminal Justice

_ Electrical Engineering

- Engineering

- Environmental Studies

- Health

- Health \& Physical Education

- Health Education

- Health Science

- History

_ Interdisciplinary Studies/Elementary

- Education

_ Management Information Systems

_ Manufacturing Engineering

- Mass Communications

- Math

_ Mechanical Engineering

- Physical Education

- Psychology

- Sociology

- Special Education

- Sport Psychology

_ Sports Management 


\section{University 17}

- Accounting
- Banking
- Business
- Business Management
- Business Marketing
- Business/Secondary Education
- Computer Information Systems
- Criminal Justice
- Education/History
- English
- Entrepreneurial Management
- Finance \& Banking
- History
- Mathematics
- Mathematics/Education
- Political Science
- Psychology
- Secondary Education/Mathematics
- Social Work

\section{University 18}

_ Accounting

- Art Education

_ Banking

- Business

Business Administration

_ Business Management

- Business Marketing

- Chemistry

- Computer Engineering

- Computer Information Sciences

- Computer Science

- Criminal Justice

- Early Childhood Education

- Economics

- Exercise Science

- Finance

_ Healthcare Management

- Information Technology

_ Justice Studies

_ Management Information Systems

- Mass Communications

- Physical Education

_ Physical Therapy

- Political Science

- Psychology

_ Rehabilitation Studies

- Sociology

- Sport Management

- Sports Medicine

- Therapeutic Recreation 
Appendix D

\section{NCAA GOALS Area of Study and Majors}

(Number in parentheses corresponds to the university code from Appendix A.)

\section{Biological Sciences (Zoology, Physiology, etc.)}

1.1. Agriculture (16)

1.2. Animal Science $(16,6)$

1.3. Biochemistry (14)

1.4. Biology $(10,11,12,13,14,15,16,2,3,4,5,7,8,9)$

1.5. Forensic Biology (1) + Forensic Science $(12,15)$

2. Business (Accounting, Marketing, Personnel, etc.)

2.1. Accounting $(10,11,14,15,16,17,18,1,2,3,4,5,6,7,8)$

2.2. Banking and Finance $(10,17,2)+$ Banking $(18,2)+$ Finance $(11,12,14,18,1,2,6,7)$

2.3. Business $(10,11,14,15,17,18,3,4,5,7)$

2.4. Business Administration (10, 11, 12, 13, 14, 15, 18, 2, 3, 4, 5, 8)

2.5. Business Economics (9)

2.6. Business Entrepreneurship (12, 2, 5) + Entrepreneurial Management (17)

2.7. Business Information Systems (10) + Information Systems (4)

2.8. Business Management $(10,12,14,16,17,18,1,2,4,5,6,8,9)+$ Healthcare Management (18)

2.9. Business Marketing $(10,11,12,14,16,18,1,2,3,4,5,7,8,9)$

\section{Communications (Journalism, Public Relations, etc.)}

3.1. Broadcast Journalism $(10,14,4,7)$

3.2. Communications $(10,11,13,15,2,4,7)+$ Communication Arts (13) + Graphic Communications Systems (6)

3.3. Journalism $(14,2,5,7)+$ Journalism and Mass Communications (6)

3.4. Mass Communications $(12,14,16,18,1,5,7,8,9)$

3.5. Media Communications (5) + Telecommunication (4)

3.6. Public Relations $(2,4)$

3.7. Speech Communications (4)

3.8. Sport Communication (9)

4. Education (Elementary, Special, etc.)

4.1. Art Education (18 - discontinued 2009)

4.2. Counselor Education (16 - graduate student)

4.3. Early Childhood Education $(10,18,9)+$ Elementary Education $(11,12,13,15,16,5,9)$

4.4. Pre-Education (1) + Education $(11,12,13,14,15,17,4,7,9)$

4.5. Health Education $(13,16,7)$

4.6. History Education $(14,6)$

4.7. Mathematics Education $(10,9,17)$

4.8. Secondary Education $(10,13,17,1)$

4.9. Special Education $(1,16)$

4.10. Technology Education (9) 


\section{Engineering, Computer / Information Sciences}

5.1. Applied Engineering Technology (6) + Engineering $(10,11,13,14,16,5,6)+$

Engineering Mathematics (15) + Engineering Technology (11)

5.2. Architecture (2) + Architectural Drafting (5) + Architectural Engineering (5) + Building

Construction Technology (5) + Construction Management (6)

5.3. Chemical Engineering $(2,6)$

5.4. Civil Engineering $(15,8)+$ Civil Engineering Technology (9)

5.5. Computer Engineering $(13,16,18,2,4,5,8)$

5.6. Computer Graphics (10) + Computer Technology (10)

5.7. Computer Information Systems $(13,15,16,17,18,7,8)+$ Information Technology (14,

$5,6)+$ Computer Information Technology $(5)$

5.8. Computer Science $(11,13,14,16,18,3,5,6,7)$

5.9. Electrical Engineering $(15,16,2,4,5,6,8)+$ Electronic Technology $(5,6)$

5.10. Industrial Engineering (4) + Industrial Engineering Technology (9) + Industrial

Technology (11)

5.11. Information Systems Engineering $(13,4)+$ Management Information Systems (16, $18,1,5,6)$

5.12. Manufacturing Engineering (16)

5.13. Mechanical Engineering $(15,16,8)+$ Mechanical Engineering Technology (9)

5.14. Optical Engineering (5)

6. Exercise, Sports, Kinesiology

6.1. Athletic Coaching Education (2)

6.2. Exercise Science $(18,2,3,5)+$ Exercise Sport Science (7) ++ Kinesiology $(2,7)+$ Kinesiotherapy (5) + Movement Science (1) + Sport Science $(11,12)+$ Sport Science and Fitness Management (6)

6.3. Fitness and Wellness (7) + Health Education (7) + Health and Physical Education $(16,2)$ + Health Fitness (5) + Health Fitness Instruction (5) + Human Performance \& Wellness (15)

6.4. Parks and Recreation Management (7) + Recreation (2) + Recreation and Tourism (2) + Recreational Sport Management (7)

6.5. Physical Education $(11,12,13,14,15,16,18,1,2,4,5,7,9)$

6.6. Sport Management $(10,11,13,14,15,16,18,1,2,7,9)$

6.7. Sports Medicine (9)

7. Humanities and Fine Arts (Music, Religion, English, etc.)

7.1. Art $(11,15,2,7)$

7.2. English $(10,11,13,14,17,2,3,4,6$

7.3. Graphic Design $(10,11,12,2)$

7.4. Music $(10,15)+$ Music Technology (10)

7.5. Theater (9) + Theater Arts (10)

7.6. Visual Arts $(10,13,14,15)$

8. Physical Sciences and Mathematics (Chemistry, etc.)

8.1. Chemistry $(11,13,15,18,1,7,8,9)+$ Organic Chemistry (2) 
8.2. Environmental Science $(14,7)+$ Environmental Studies (16)

8.3. Geology (12)

8.4. Mathematics $(10,11,13,15,16,17,4,5,6,7,8)$

8.5. Physical Science (15)

8.6. Physics $(14,1,7)$

9. Professional Studies (Nursing, Occupational Therapy, etc.)

9.1. Athletic Training $(15,7)$

9.2. Community Health $(13,1)+$ Public Health Education (7)

9.3. Counseling (2)

9.4. Health Science $(14,16,9)$

9.5. Hospitality Management (5) + Hospitality and Tourism (7) + Healthcare Management (18)

9.6. Medical Technology (4)

9.7. Nursing $(10,5,7,9)+$ Pre-Nursing (12)

9.8. Pharmaceutical Science (7) + Pharmacy $(11,2)$

9.9. Physical Therapy $(11,14,18,1,7)+$ Therapeutic Recreation (18) + Recreational

Therapy (2) + Rehabilitation Studies (18)

9.10. $\quad$ Law (7)

9.11. Pre-Medicine $(11,12,7)$

9.12. Public Administration (10)

9.13. Social Work $(10,17,1,2,3,6,7)$

10. Social Sciences (Psychology, History, Economics, etc.)

10.1. Anthropology (14)

10.2. Behavior Analysis (8) + Child Development (9) + Pedology (10) + Family and Co5mer Science (9) + Human Ecology (3)

10.3. Criminal Justice $(10,11,12,13,14,15,16,17,18,1,2,3,4,5,6,7,8,9)+$ Criminology $(13,2)+$ Justice Studies (18)

10.4. Economics (18)

10.5. Geography (12)

10.6. History $(10,11,13,14,16,17,2,5,7,9)+$ Military History (5)

10.7. Political Science $(10,11,14,15,17,18,2,5,6,8,9)$

10.8. Psychology $(10,11,12,13,15,16,17,18,1,2,4,5,6,7)$

10.9. Social Science $(5,7)+$ Sociology $(10,11,12,14,15,16,18,2,3,4,5,7,9)$

\section{Other Academic Field}

11.1. Aviation Science (11) + Aviation (2)

11.2. Fire Science (12) + Science (2)

11.3. General Studies $(11,15,3)$

11.4. Integrated Studies (1)

11.5. Interdisciplinary Studies (5)

11.6. Liberal Studies $(15,6)+$ Liberal Arts (2) 